\title{
Defect States at Organic-Inorganic Interfaces: Insight from First Principles Calculations for Pentaerythritol Tetranitrate on MgO Surface
}

\author{
Roman V. Tsyshevsky ${ }^{+}$, Sergey N. Rashkeev ${ }^{++}$, and Maija M. Kuklja ${ }^{+1}$ \\ ${ }^{+}$Department of Materials Science and Engineering, University of Maryland, College Park, MD 20742 \\ ${ }^{\ddagger}$ Qatar Environment \& Energy Research Institute, P.O. Box 5825, Doha, Qatar
}

\begin{abstract}
Light-responsive organic-inorganic interfaces offer experimental opportunities that are otherwise difficult to achieve. Since laser light can be manipulated very precisely, it becomes possible to engineer selective, predictive, and highly controlled interface properties. Photochemistry of organic-inorganic energetic interfaces is a rapidly emerging research field in which energy absorption and interface stability mechanisms have yet to be established. To explore the interaction of the laser irradiation with molecular materials, we performed first principles calculations of a prototype organic-inorganic interface between a nitroester (pentaerythritol tetranitrate, PETN, $\mathrm{C}_{5} \mathrm{H}_{8} \mathrm{~N}_{4} \mathrm{O}_{12}$ ) and a magnesium oxide ( $\mathrm{MgO}$ ) surface. We found that the light absorption is defined by the band alignment between interface components and interfacial charge transfer coupled with electronic states in the band gap, generated by oxide surface defects. Hence the choice of an oxide substrate and its morphology makes the optical absorption tunable and governs both the energy accumulation and energy release at the interface. The obtained results offer a possible consistent interpretation of experiments on selective laser initiation of energetic materials, which reported that the presence of metal oxide additives triggered the photoinitiation by an excitation energy much lower than the band gap. We suggest that PETN photodecomposition is catalyzed by oxygen vacancies ( $\mathrm{F}^{0}$ centers) at the $\mathrm{MgO}$ surface. Our conclusions predict ways for a complete separation of thermo- and photo-stimulated interface chemistry of molecular materials, which is imperative for highly controllable fast decomposition and was not attainable before. The methodology described here can be applied to any type of molecular material/wide band gap dielectric interfaces. It provides a solid basis for novel design and targeted improvements of organic-inorganic interfaces with desired properties that promise to enable vastly new concepts of energy storage and conversion, photocatalysis, and molecular electronics.
\end{abstract}

Keywords: charge transfer, oxygen vacancy, F center, structural and electronic defects, excited states, hybrid functionals, molecular energetic materials, nitro compounds, oxides, nanostructures, optical absorption, photoconversion

\footnotetext{
${ }^{1}$ Corresponding author, mkukla@nsf.gov with cc mkukla@umd.edu
} 


\section{INTRODUCTION}

Understanding of energy release processes in molecular energetic materials [1] is of great interest to scientists and engineers because of a wide array of applications ranging from medicine to rocket engine fuels to planetary and environmental sciences. These applications include, for example, design of new materials [2] with targeted superior properties (e.g., superhard or superdense materials) [3,4] for materials research and new pharmaceuticals $[5,6]$ for medicine, developments of safe and efficient explosives and oxidizers for railroad, construction, and coal mining industries, novel ways to increase the energy density of solid fuels and propellants for energy research and space programs, improvements of explosive sensors and detectors for combat against terrorism $[7,8]$, and innovations in handling and recycling of toxic explosives for sustainable and clean environment [9].

The laser irradiation is promising as it provides an opportunity for selective, predictive and controlled initiation of the energy release in energetic materials. However, unlike photochemistry of biomolecules [10] or gas molecules [11,12], which is now a relatively well-established field, light-induced chemistry of surfaces or organic-inorganic interfaces involving energetic molecules is a rapidly emerging area of research with many unresolved questions $[13,14,15,16,17,18]$. Although the possibility of photolysis of energetic materials has been long realized [19,20], a useful method has only recently been developed [21,22] because most energetic materials are transparent to visible light and cannot be decomposed directly, but only by radiation with wavelengths shorter than $190 \mathrm{~nm}$ [23,24]. Understanding of individual photoinduced processes in relation to specific interface structures [25] and knowledge of mechanisms of energy accumulation and release [26] remain among outstanding challenges that hamper advances in new technologies and novel applications of engineered interfaces. Here we show that the electronic structure and optical properties of an energetic molecule adsorbed on an oxide surface or on a surface defect significantly differ from the properties of individual components and those changes can be effectively manipulated to enable control of both the energy absorption and release at the interface.

As a model organic-inorganic interface system for our study we selected pentaerythritol tetranitrate $\left(\mathrm{C}_{5} \mathrm{H}_{8} \mathrm{~N}_{4} \mathrm{O}_{12}\right.$, also known as PETN) $[27,28,29]$, a nitrate ester, which is usually used as an energetic material and a pharmaceutical (related to nitroglycerin), and $\mathrm{MgO}$, an important metal oxide [30,31,32,33,34,35,36,37,38,39], known for multifunctional properties including 
superb adsorption [40]. Both materials are being thoroughly investigated individually and hence there is a great deal of data available for validation. In addition, recent experiments on selective photoinitiation [21,22] of PETN crystals mixed with $\mathrm{MgO}$ additives suggested that decomposition of PETN is triggered by a laser-induced optical excitation with an unusually low activation energy of $1.17 \mathrm{eV}(1060 \mathrm{~nm})$ [41,42]. At a glance, it appears puzzling how the mixture of two wide-gap dielectric materials, such as PETN [43] and $\mathrm{MgO}[44,45,46,47,48,49,50]$, can absorb photons of such a low energy to initiate decomposition.

This paper is aimed at addressing three fundamental questions: ( $i$ ) what is a mechanism of the low energy photon absorption at the organic-inorganic interface, (ii) what factors govern how the electronic structure and optical properties of the interface differ from those of individual materials, and (iii) whether the light absorption and energy release can be manipulated by the chemical or structural composition of the interface. The quantum-chemical study is performed by means of density functional theory (DFT) with hybrid density functionals and large periodic supercells. With the illustrative example of the PETN - MgO model, which serves in our research as an interface prototype, we propose a possible mechanism of the optical absorption of the organic-inorganic interface and found that preexisting oxide surface defects (e.g., F - centers in oxides) generate local electronic states within the band gap of the interface. Transitions from these states enable the laser light absorption at long wavelengths and effectively allow for a modulation of the band gap of the interface by variations in chemical composition and morphology of the surface. This, in turn, governs both the energy absorption and release at the interface.

\section{COMPUTATIONAL APPROACH}

First-principles calculations were performed by employing density functional theory [51,52] (DFT) with exchange gradient-corrected correlation functional of Perdew, Burke and Ernzerhof (PBE) [53] and its hybrid version PBE0 [54] as implemented in the VASP code [55,56,57]. The projector augmented-wave (PAW) pseudo-potentials [58] were used. The structures of a PETN molecule, an ideal $\mathrm{MgO}$ crystal, and the $\mathrm{MgO}$ surfaces with and without vacancies were relaxed and compared to available experimental data and previous theoretical studies.

Atomic coordinates were relaxed by using the PBE functional without any symmetry constraints. The quality of this relaxation is illustrated for PETN in Table 1. To correct the 
significantly underestimated band gap energies, obtained from PBE, a self-consistent single point calculation was performed for each configuration by using hybrid PBE0 functional. Such an approximation improves the band gaps making them very close to experimental excitation energies.

Table 1. The lattice parameters of the PETN crystal, the parameter $\varepsilon$ measures accuracy of calculations vs experimental data.

\begin{tabular}{|c|c|c|c|c|c|c|}
\hline \hline Method & \multicolumn{2}{|c|}{$\boldsymbol{a}(\AA)$} & $\boldsymbol{\varepsilon}(\boldsymbol{\%})$ & \multicolumn{1}{c|}{$\boldsymbol{b}(\AA)$} & $\boldsymbol{\varepsilon}(\boldsymbol{\%})$ & \multicolumn{2}{c|}{$\boldsymbol{c}(\AA)$} & \multicolumn{1}{c|}{$\boldsymbol{\varepsilon}(\boldsymbol{\%})$} \\
\hline \hline PBE & 9.64 & 2.8 & 9.64 & 2.8 & 6.82 & 1.6 \\
\hline PBE0 & 9.37 & 0.1 & 9.38 & 0.0 & 6.79 & 1.2 \\
\hline Exp.[59] & 9.38 & - & 9.38 & - & 6.71 & - \\
\hline \hline
\end{tabular}

In calculations of an ideal $\mathrm{MgO}$ crystal, the convergence criterion for total energy was set to $10^{-5} \mathrm{eV}$, and the maximum force acting on each atom in the periodic cell was set not to exceed $0.01 \mathrm{eV} / \AA$. In most of calculations, we used $3 \times 3 \times 3$ Monkhorst-Pack $\boldsymbol{k}$-point mesh, and the kinetic energy cut-off was set to $520 \mathrm{eV}$. The calculated lattice constant of the cubic (rock salt) unit cell, $\boldsymbol{a}=4.256 \AA$, agrees with the experimental lattice vectors of $\boldsymbol{a}=4.216 \AA$ [44] within $1 \%$. To simulate the $\mathrm{MgO}$ surface we used a periodic slab model. A rectangular block of $8 \times 8 \times 4$ ions represented a supercell, which consists of the four-layer $\mathrm{MgO}$ slab cut from the bulk $\mathrm{MgO}$ structure to form the $\mathrm{MgO}$ surface with the (001) orientation, with the supercell lattice vectors of $\boldsymbol{a}=\boldsymbol{b}=16.57 \AA$ and $\boldsymbol{c}=20.0 \AA$. The vacuum layer of $\sim 14 \AA$ placed on top of the (001) $\mathrm{MgO}$ surface was intended to minimize interactions between supercells in $z$ direction and to ensure that electronic states of different slabs do not overlap.

To explore the electronic structure of the system, which consists of the PETN molecule adsorbed on the (001) $\mathrm{MgO}$ surface, the vacuum layer thickness was increased up to $20 \AA$. Our test convergence calculations demonstrated that the $20 \AA$ thickness of the vacuum layer is sufficient for the accurate evaluation of total energies and geometry parameters of the absorbed PETN molecule. The further increase beyond $20 \AA$ does not have any significant effect on the studied parameters of the system. The lattice vectors of the corresponding supercell used in calculations were $\boldsymbol{a}=\boldsymbol{b}=16.57 \AA$ and $\boldsymbol{c}=26.0 \AA$. 
It is now common practice to include (at least semi-empirically) the van der Waals interaction into calculations of organic molecules at insulating surfaces [60,61]. Because most of our calculations were performed for an individual PETN molecule adsorbed on the MgO surface, with only one or two atoms of the molecule forming bonds with atoms at the oxide surface, we assumed that the van der Waals interactions can be neglected as they should not affect the electronic structure and optical transitions of our system in a significant way. Nevertheless, we performed a series of test calculations to estimate the effect of the van der Waals interactions on the electronic structure of PETN-MgO interface by using Grimme's PBE-D2 [62] functional and vDW-DF $[63,64,65]$ functional of Langreth, Lundqvist et al (Quantum Espresso program suite [66]).

Decomposition reactions of the PETN gas-phase molecule and its anion radical were carried out using Becke three parameter hybrid B3LYP [67] functional with 6-31+G(d,p) basis set within Gaussian 09 [68] program.

\section{RESULTS AND DISCUSSION}

\subsection{The Structural Model}

Ideally, a comprehensive investigation of optical properties of the PETN-MgO composite system should include simulations of various possible interfaces formed by PETN and $\mathrm{MgO}$ semi-infinite crystals. To be realistic, a consideration of all possibilities would require significant computational resources and, most importantly, a detailed knowledge of all the interface structures, which is not readily available for the time being. Hence, as the first step in the investigation of the electronic and optical properties of the PETN-MgO system, we constructed a simplified model, which consists of one PETN molecule placed on the (001) MgO surface (Fig. 1). Since the electronic structure of organic molecular crystals is fully defined by the constituting molecules, such a model will serve as a good starting point and should be able to provide a useful insight on the behavior of crystal interfaces. A similar model has been used to investigate interactions of various nitro-containing molecules with silicon [69], aluminum [70] and aluminum oxide [71] surfaces.

The $\mathrm{MgO}$ supercell was cut from the fully relaxed $\mathrm{MgO}$ rock salt crystalline structure to expose the (001) surface (Fig. 2a). The relaxation and rumpling at the $\mathrm{MgO}(001)$ surface are small. The shift of the surface plane as a whole does not exceed $1 \%$ of the lattice constant. In 
addition to the perfect (defect-free) surface, we explored $\mathrm{MgO}$ surfaces containing the most common single defect in oxides, a neutral oxygen vacancy (F-center).

The PETN molecule (Fig. 3) was subsequently placed on an ideal (001) $\mathrm{MgO}$ surface or on a defect-containing surface (Fig. 1a-d). PETN crystals primarily expose the (101) and (110) low energy facets [72, see also discussions in 73 and 74], which are illustrated in Fig. S1 in Supplementary Materials. On the (101) surface, PETN molecules are positioned in such a way that one $\mathrm{C}-\mathrm{CH}_{2}-\mathrm{O}-\mathrm{NO}_{2}$ tail is directed almost perpendicualar to the surface (Fig. 1b). This configuration of the PETN molecule was, therefore, chosen for our study to be adsorbed on $\mathrm{MgO}$ as a simplified model of the interface between $\mathrm{MgO}$ (001) and PETN (101) surfaces (Fig. 1c). The most stable position, obtained by the full relaxation, corresponds to the structure in which: (i) both oxygen atoms of the nitro group attach to Mg atoms at the oxide surface, and (ii) the PETN oxygen atoms are located at distances of 2.278 and $2.725 \AA$, respectively, above the surface magnesium atoms (Fig. 1c). It is quite natural that $\mathrm{O}$ atoms of the $\mathrm{NO}_{2}$ group with an excessive negative charge are attracted to $\mathrm{Mg}$ atoms at the surface with some excess of the positive charge. And this was the case when the energy gets its minimum. The starting configuration could be any configuration with only one $\mathrm{NO}_{2}$ group getting the position closest to the $\mathrm{MgO}$ surface with the subsequest rotation of the molecule around the axis perpendicular to the surface. Although several different metastable configurations may be formed in such a way, the lowest energy configuration, corresponding to the structure described above, was used in our research. 

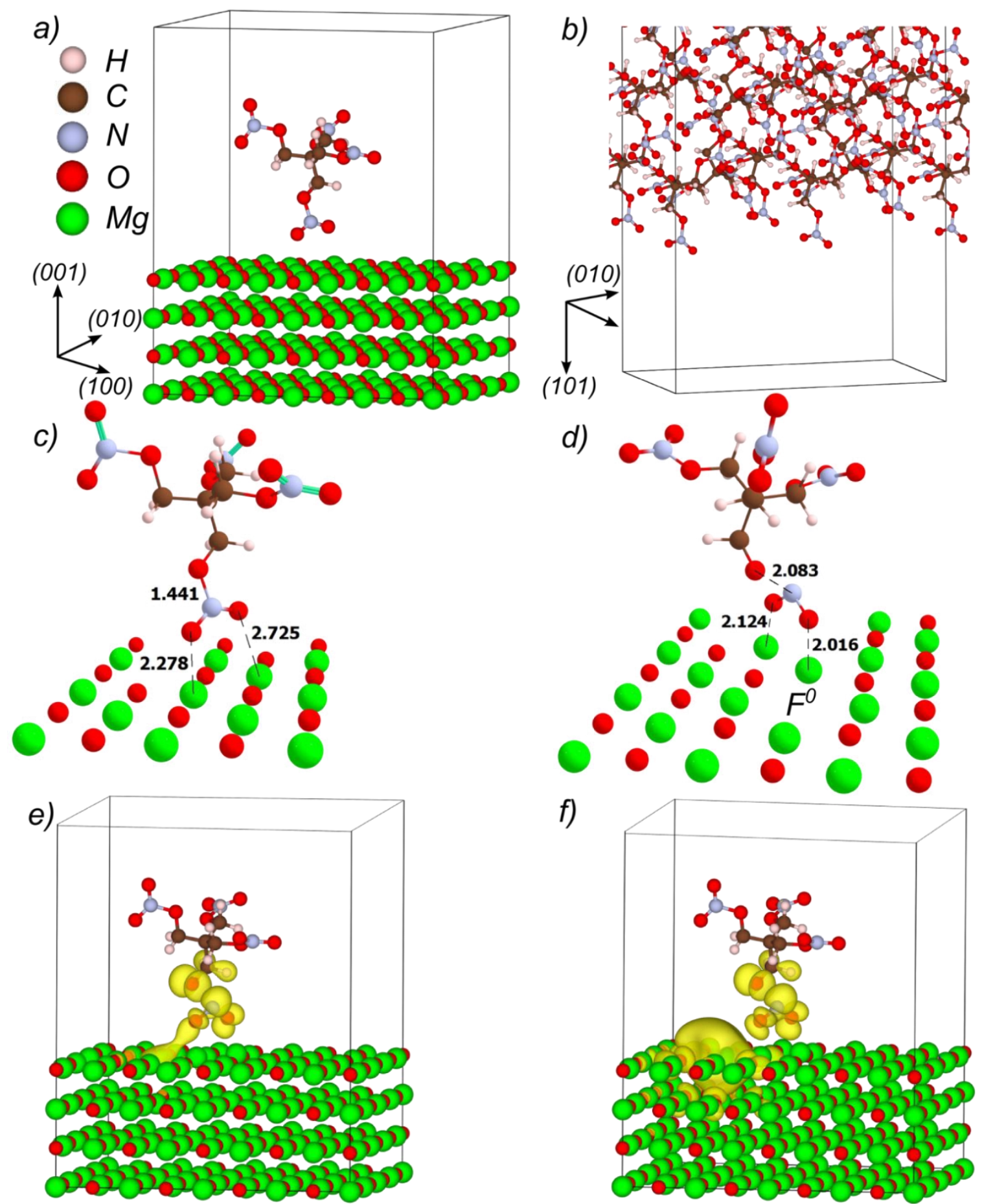

HOMO

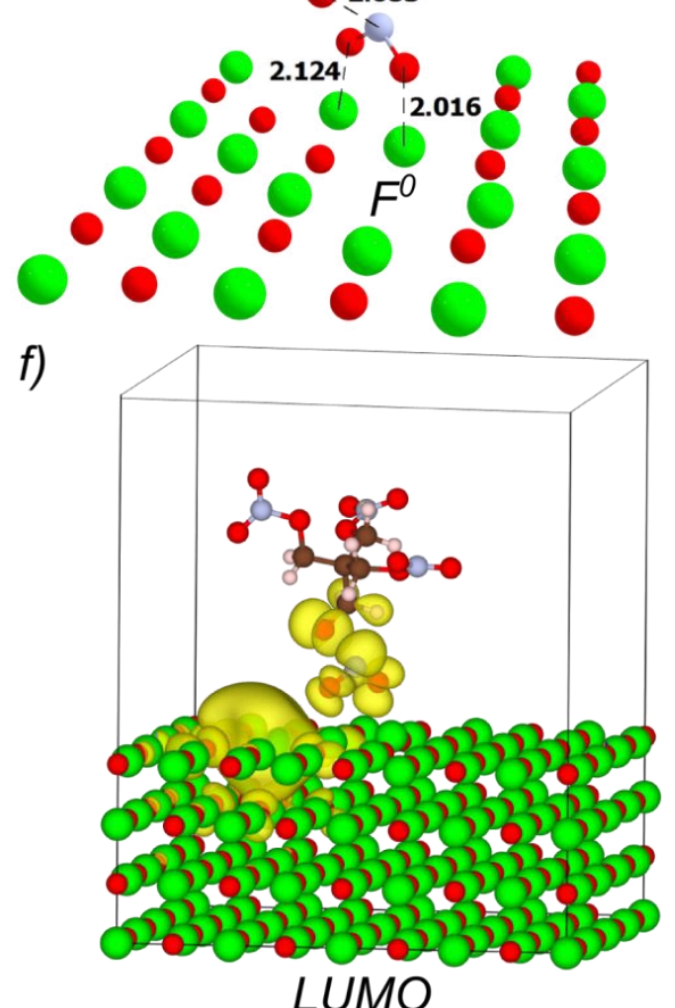

Figure 1. a) The structure of the model supercell representing a PETN molecule adsorbed on the (001) $\mathrm{MgO}$ surface is shown. b) The configuration of the PETN molecule corresponds to its position on the (101) PETN surface a fragment of which is demonstrated. The atomic structures are illustrated for the PETN molecule adsorbed on c) the ideal (001) $\mathrm{MgO}$ surface and d) the surface containing an $\mathrm{F}^{0}$-center. HOMO (e) and LUMO (f) of the PETN molecule adsorbed atop the $\mathrm{F}^{0}$-center on $\mathrm{MgO}$ are depicted. All considered configurations were fully optimized. 


\subsection{The Electronic Structure of $\mathrm{MgO}$ (001) Surface Containing Oxygen Vacancies}

We started with modeling of an ideal (001) $\mathrm{MgO}$ surface. The electronic structure of both bulk and surface magnesium oxide has been previously studied in great detail theoretically and experimentally [30-39].

We found that the top of the $\mathrm{MgO}$ valence band is formed by $2 p$ functions of oxygen (Fig. $2 b)$, in agreement with previous studies [32,33,75]. The width of this oxygen band is $4.87 \mathrm{eV}$. The electronic structure of $\mathrm{MgO}$ is characterized by strong localization of valence electrons and a large band gap between occupied anion $(\mathrm{O} 2 p)$ and unoccupied cation $(\mathrm{Mg} 3 s)$ states.

To reproduce the experimental value of the energy gap of the ideal bulk $\mathrm{MgO}$ crystal, three simple approximations were used: $(i)$ both the lattice relaxation and the electronic structure calculations were performed with PBE, (ii) the lattice relaxation was performed with PBE while the electronic structure was refined with hybrid PBE0, and (iii) the lattice configuration was taken from experiment and the electronic structure was calculated with hybrid PBE0. As expected, the band gap energy of the super cell fully relaxed with PBE, $4.45 \mathrm{eV}$ (Table 2), is $\sim 40 \%$ underestimated with respect to its experimental value of $7.78 \mathrm{eV}$ [44]. The hybrid PBE0 functional calculation yields $7.21 \mathrm{eV}$, which is much closer to the experiment, when the experimental lattice parameters of $\boldsymbol{a}=4.216 \AA$ [44] are imposed and no additional relaxation with PBE0 is allowed. The same approach gives even closer value of $7.50 \mathrm{eV}$ when the lattice constant of $\boldsymbol{a}=4.210 \AA$ [76,77] taken from another experimental reference is used. Once the electronic structure calculation is repeated with the hybrid PBE0 functional for the geometry configuration that was optimized with PBE, the band gap, $6.91 \mathrm{eV}$, becomes slightly ( 10\%) underestimated but still is in reasonable agreement with experiment and provides a better agreement with experiment than PBE alone. Hence, to reduce the GGA error in the band gap predictions and yet to allow for the lattice configuration relaxation, which is extremely important in our further study, we use the computational strategy in which the structure is fully relaxed with PBE while the band gap is further refined with hybrid functional PBE0. This practical approach offers a reasonable compromise between affordable computational resources and the required accuracy of the calculated electronic excitations.

Next, the calculated band gap of the $\mathrm{MgO}(001)$ surface is reduced to $5.72 \mathrm{eV}$ relative to the bulk value of $6.91 \mathrm{eV}$ (Table 2, Fig. 2), which is in respectful agreement (within 7\%) with the 
high-resolution electron energy loss spectra of $\mathrm{MgO}$ that have demonstrated that the exciton absorption peak shifts from $7.78 \mathrm{eV}$ in the bulk [44,45] to $6.15 \mathrm{eV}$ at the (001) surface plane [78]. Interestingly, low coordinated sites at the surface give a range of lower excitation energies, 4.5-5.5 eV [45-50], and rough $\mathrm{MgO}$ surfaces and powders were observed to be sensitive even to low-fluence radiation with photon energies as low as $3.5 \mathrm{eV}$ [48].

Further, the oxygen vacancy formation energy, $\Delta E_{(\mathrm{Vo})}$ was calculated with respect to the atomic oxygen in its ground state, $\Delta E^{l}{ }_{(\mathrm{Vo})}=E_{\mathrm{V}_{\mathrm{o}}}-\left[E_{0}+\mathrm{E}(\mathrm{O})\right]$, where $E_{\mathrm{V}_{\mathrm{o}}}$ is the energy of $\mathrm{MgO}$ containing an oxygen vacancy, $\mathrm{E}_{0}$ is the energy of the ideal $\mathrm{MgO}$, and $\mathrm{E}(\mathrm{O})$ is the energy of the oxygen atom. The $\Delta E_{(\mathrm{Vo})}^{l}$ of $9.17 \mathrm{eV}$ is found to be in agreement with earlier estimates of 9.07 $\mathrm{eV}$, obtained from the embedded cluster model [32], and $9.02 \mathrm{eV}$, obtained from periodic calculations [79]. Consequently, the $\Delta E_{\left({ }^{2}\right)}^{2}$ was calculated using the standard expression, $\Delta E_{(\mathrm{Vo})}^{2}=E_{\mathrm{Vo}}-\left[E_{0}+1 / 2 \mathrm{E}\left(\mathrm{O}_{2}\right)\right]$, where $\mathrm{E}\left(\mathrm{O}_{2}\right)$ is the energy of the free oxygen molecule in its triplet state. The vacancy formation energy $\Delta E_{(\mathrm{Vo})}^{2}$ happened to be $6.15 \mathrm{eV}$, in reasonable agreement with the corresponding estimate of $5.83 \mathrm{eV}$, also obtained from periodic calculations [80].

Charge density in the vacancy is $-1.5 e$, showing that almost two electrons are trapped in the vacancy with a small portion of it delocalized over nearest $\mathrm{Mg}$ ions. The optimized geometry of the $\mathrm{F}^{0}$-center at the surface shows only a small relaxation (1.6\% of the lattice constant) of the nearest $\mathrm{Mg}$ ions outwards the vacancy and even smaller displacements (1\% of the lattice constant) perpendicular to the surface direction. The relaxation energy is also small and does not exceed $0.12 \mathrm{eV}$, which is consistent with the fact that the defect is neutral with respect to the crystalline lattice. The observed trend is consistent with the results of previous theoretical studies [32-34,81].

The $\mathrm{F}^{0}$-center on the (001) $\mathrm{MgO}$ surface generates local electronic states in the band gap at $3.07 \mathrm{eV}$ above the top of the valence band, in agreement with $3.00 \mathrm{eV}$ obtained in previous calculations [32]. The relative energy positions of the $\mathrm{MgO}$ bulk, ideal surface, and the surface with oxygen vacancy are shown in Fig. $2 \mathrm{c}$ and the isosurface of the $\mathrm{F}^{0}$-center electronic function is depicted in Fig. 2b. 
Table 2. Calculated band gaps $\mathbf{E}_{\text {gap }}(\mathrm{eV})$ in model systems.

\begin{tabular}{lll}
\hline \hline \multirow{2}{*}{ Model } & \multicolumn{2}{c}{$\mathbf{E}_{\text {gap }}$} \\
\cline { 2 - 3 } & PBE $^{\mathrm{a}}$ & PBE0 $^{\mathrm{b}}$ \\
\hline \hline MgO & & \\
Ideal Bulk Crystal & 4.45 & $6.91(7.21)^{\mathrm{c}}$ \\
Ideal (001) Surface & 3.67 & 5.72 \\
$\mathrm{~F}^{0}$ center on (001) Surface & 1.40 & 2.65 \\
\hline \hline PETN & & \\
Isolated Molecule & 4.36 & $7.00[7.32]^{\mathrm{d}}$ \\
Ideal Bulk Crystal & 4.09 & $6.84[7.07]^{\mathrm{d}}$ \\
Ideal (101) surface & 3.85 & 6.54 \\
\hline \hline PETN adsorbed atop & & \\
Ideal (001) MgO Surface & $1.22(1.27,1.19)^{\mathrm{e}}$ & 3.42 \\
$\mathrm{~F}^{0}$ center on (001) MgO Surface & 0.40 & 1.32 \\
\hline \hline
\end{tabular}

${ }^{a}$ The PBE underestimate the band gaps about $40 \%$ and are given here for comparison

${ }^{b}$ The energy gap was refined with PBE0 functional while the structural parameters were optimized with PBE

${ }^{c}$ The band gap was calculated using the experimental crystallographic parameters of the bulk $\mathrm{MgO}$ crystal

${ }^{d}$ The energy gap was calculated using PBE0 functional with the full geometry relaxation

${ }^{e}$ The energies in parentheses are corrected for van der Waals interactions and are obtained with PBE-D2 and VDWDF functionals, respectively 

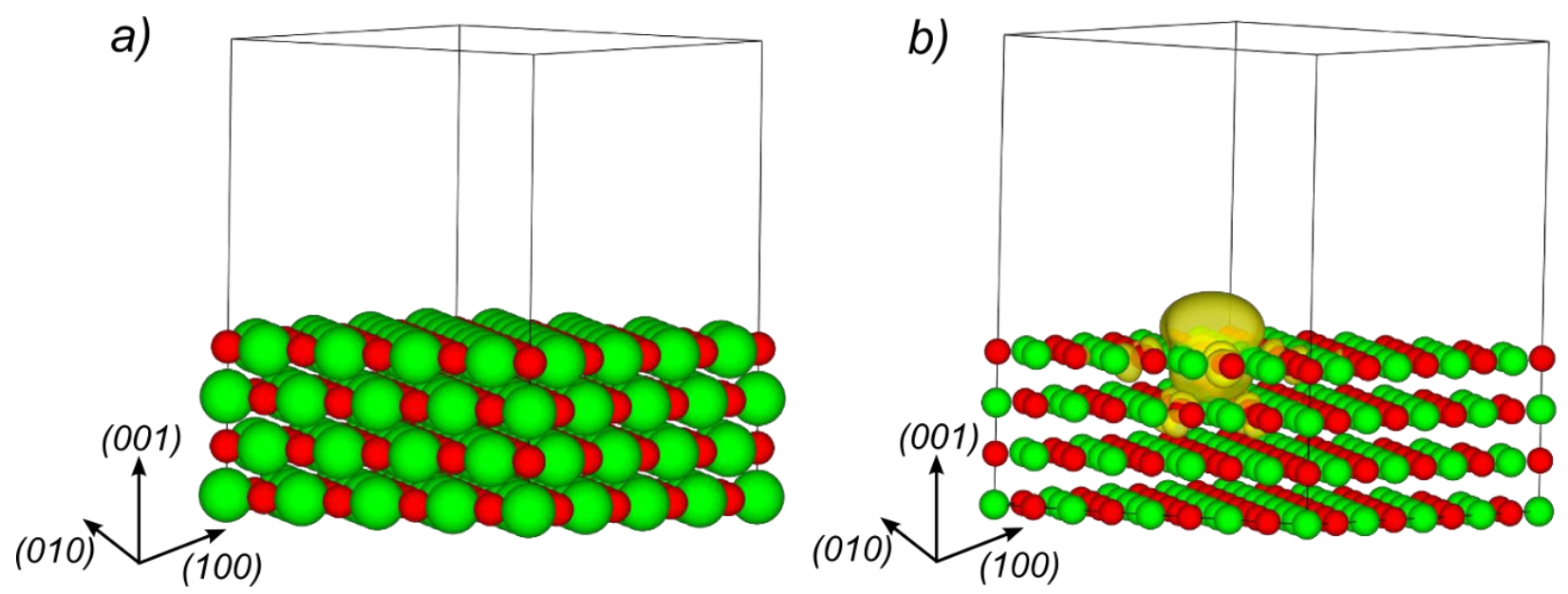

c)

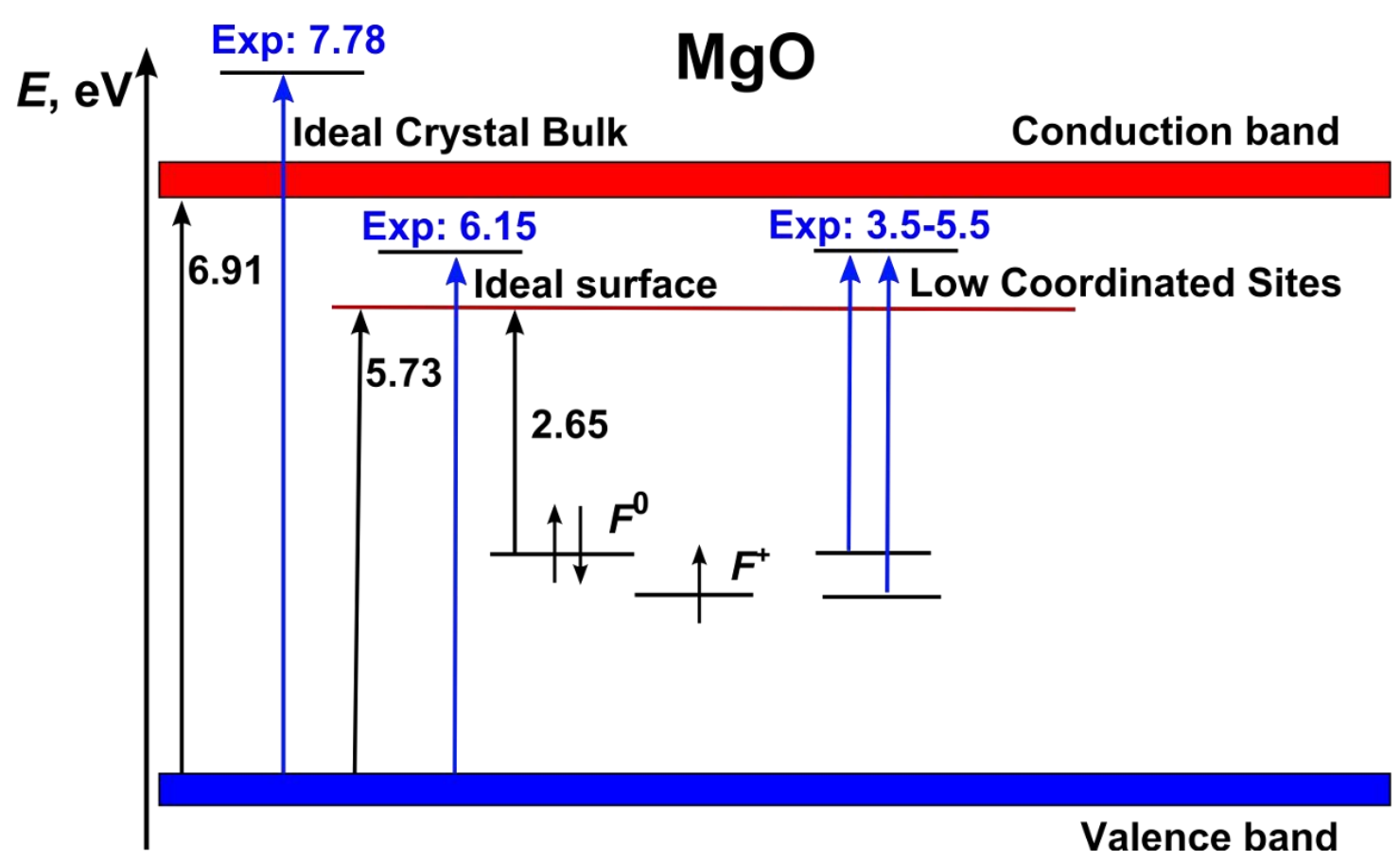

Figure 2. a) A model supercell of the (001) MgO surface; b) Decomposed charge density of the highest occupied crystalline orbital of the (001) $\mathrm{MgO}$ surface containing an F-center; c) A diagram showing the relative energies of F-center states on the (001) surface of $\mathrm{MgO}$ versus the bulk and surface band gaps. The calculated energies are shown in black and experimental measurements are indicated in blue.

\subsection{Molecular and Crystalline PETN}

With only a few quantum-chemical studies that touched upon the electronic structure of crystalline PETN [29,73], the nature of experimental optical properties of the material [27,28] was barely understood until recently [43]. The atomic structures of the PETN molecule and the 
crystal with the $P-42_{1} c$ space group and two molecules per unit cell are shown in Figs. 3a and $3 \mathrm{~b}$. The calculated lattice constants of the PETN crystal obtained with the hybrid PBE0 are appreciably closer to the experimental X-ray diffraction measurements of $\boldsymbol{a}=\boldsymbol{b}=9.38 \AA, \boldsymbol{c}=6.71 \AA$ [59] than the vectors obtained from PBE (Table 1).

In molecular calculations [43], the PETN HOMO is found to be a non-bonding orbital formed predominantly by $p$ functions of oxygen atoms from nitro groups (Fig. 3c). The full relaxation of the isolated PETN molecule causes the four - $-\mathrm{NO}_{2}$ tails to have slightly different bond lengths and torsion angles, which is reflected in the splitting of the four occupied molecular orbitals. The quasi-degenerate HOMO-1, HOMO-2 and HOMO-3 are also non-bonding orbitals of similar nature and lie only slightly below HOMO.

The LUMO is an anti-bonding $\pi^{*}$ molecular orbital (Fig. 3d) with a large contribution of $p$ functions of nitrogen and oxygen atoms. Similarly to the occupied orbitals, LUMO is followed by quasi-degenerate $\mathrm{LUMO}+1, \mathrm{LUMO}+2$ and $\mathrm{LUMO}+3$ with the energy only slightly higher than LUMO.

Periodic calculations give similar results for PETN crystals. The top of the valence band of crystalline PETN is formed by a combination of $2 p$ states of oxygen atoms, and the bottom of the conduction band is predominantly made of $2 p$ states of oxygen and nitrogen atoms from nitro groups, as shown in Fig. 3 for molecules. Isosurfaces of the top of the valence band and the bottom of the conduction band of an ideal PETN crystal resemble the corresponding HOMO and LUMO isosurfaces of a single PETN molecule. This, indeed, should be expected from the nature of molecular crystals in which all electronic density is well localized on molecules.

Table 2 shows that the calculated HOMO-LUMO gap of the PETN molecule, $4.36 \mathrm{eV}$, obtained with PBE, is $\sim 40 \%$ smaller than the value of $7.32 \mathrm{eV}$, produced with PBE0. The HOMO-LUMO gap corrected by hybrid PBE0 in the molecular configuration fully relaxed with PBE is $7.00 \mathrm{eV}$. This obvious correspondence of the energy gaps, $7.32 \mathrm{eV}$ that is fully obtained with PBE0 and $7.30 \mathrm{eV}$ that is relaxed with PBE and refined with PBE0, serves as another illustration of reliability of the chosen approach. The data is also consistent with $6.64 \mathrm{eV}$ calculated with B3LYP [43].

PETN band gaps obtained from the periodic calculations, shown in Table 2, follow the same trend as the molecular HOMO-LUMO energies, but are systematically lower, as should be expected due to weak intermolecular interactions in molecular crystals. In particular, the band 
gap of $4.09 \mathrm{eV}$ (PBE) agrees well with previous calculations (4.2 eV) [29] and is visibly lower than the obtained using hybrid PBE0 $7.07 \mathrm{eV}$ (Table 2, see also discussion in [43]) while the combined PBE/PBE0 computational scheme delivers $6.84 \mathrm{eV}$. Unsurprisingly, the PETN surface calculations yield 3.85 (with PBE) and $6.54 \mathrm{eV}$ (with PBE0), respectively, showing slightly reduced values as compared to both the isolated molecule and bulk crystal results (Table 2). A small difference in the energy gaps between the PETN molecule and the ideal crystal (as well as between the PETN molecule and the ideal surface) coupled with the observed localization of molecular (and crystalline) orbitals on individual molecules lend an additional support to the structural model we chosen for the PETN-MgO interface. Hence the use of a single PETN molecule adsorbed on an $\mathrm{MgO}$ surface should be sufficiently illuminating to elucidate the nature of the low energy optical transitions in the PETN-MgO system.

The most important observation here is that both the HOMO-LUMO transitions in the PETN molecule and the band gap transitions in the PETN crystal are localized on $\mathrm{NO}_{2}$ groups. Their energies however are fairly high, 6.54-7.32 eV, and thus they are inconsistent with electronic excitations triggered by $1.17 \mathrm{eV}$ laser excitation [22]. This discrepancy essentially rules out optical transitions fully localized on PETN to play a role and leads us to believe that the absorption observed in experiments [22] should be relevant to direct interactions between the oxide surface and PETN, for example, to a possible charge transfer prompted by $\mathrm{MgO}$ additives. 


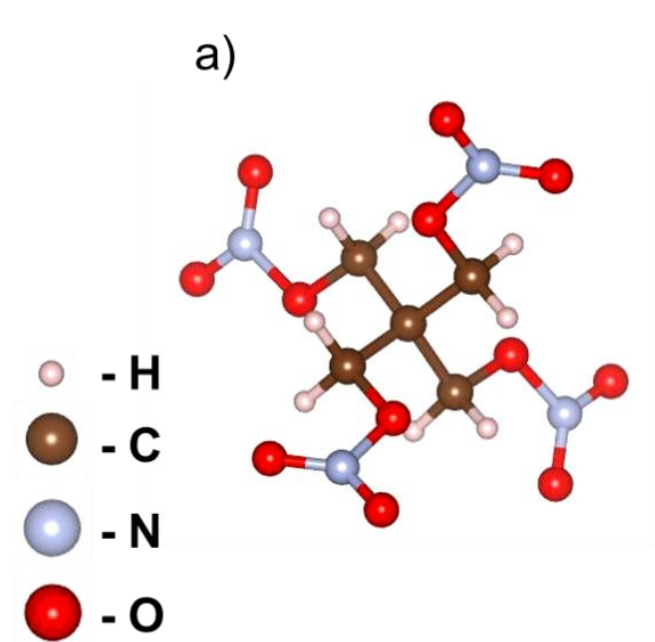

c)

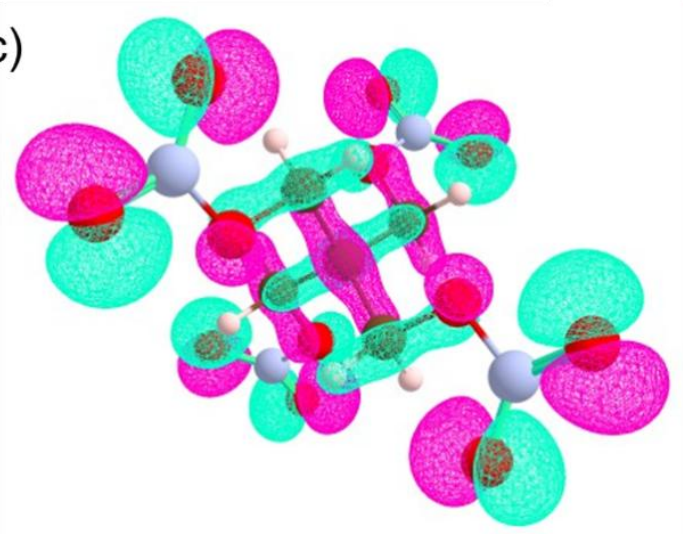

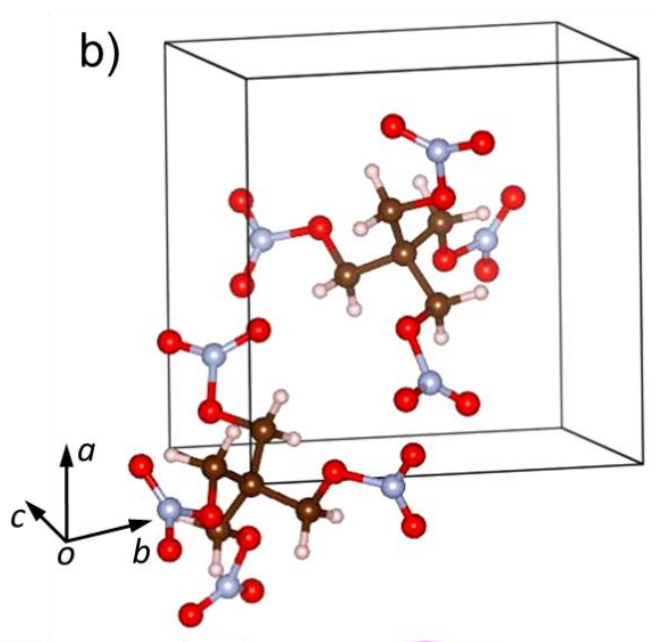

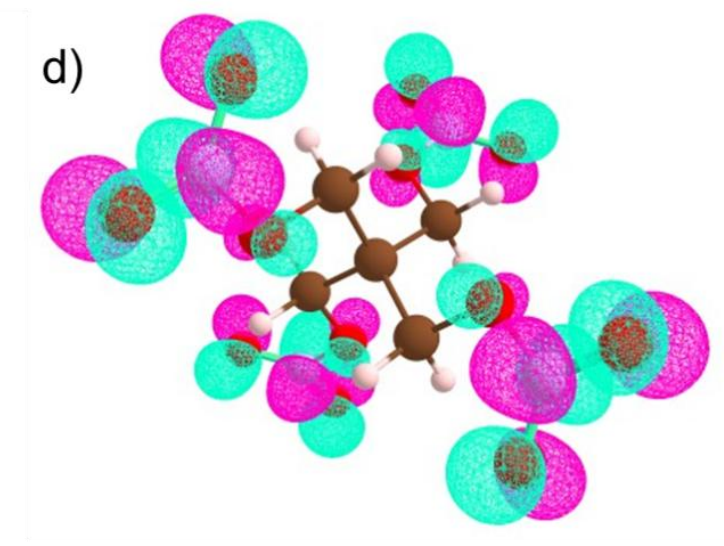

Figure 3. a) The molecular and b) the crystalline PETN structure; c) the electron density distribution of the molecular PETN HOMO state and d) the LUMO (isosurfaces corresponding to coefficients with a positive sign are colored in magenta, and with a negative sign are shown in cyan).

\subsection{Band Discontinuities at Interfaces}

The relative positions of valence and conduction bands at the MgO-PETN interface are analyzed here from the point of view of band alignment of isolated $\mathrm{MgO}$ and PETN components. While the physics of the Schottky barrier (a potential energy barrier for electrons formed at a metal-semiconductor junction) is relatively well understood, much less is known about the band alignment at the insulator-insulator interfaces, such as a wide band gap nitroester PETN - wide band gap oxide $\mathrm{MgO}$ interface. The Schottky rule for the band alignment at a metalsemiconductor heterojunction says that the discontinuity of the energy levels at the metalsemiconductor interface is a difference between the metal work function and the electron affinity of the semiconductor [82]. This simple recipe was very successful, and most of the later theories provided only small corrections to the so-called Schottky limit $[83,84]$. 
As a DFT method is employed, the unoccupied and excited states are not calculated with required accuracy. Hence, only the valence band discontinuity can be accurately modeled. To calculate the valence band offsets, two different methods could be used. The first one is the reference potential method [85]. As the reference energy, one should use the macroscopically averaged electrostatic potential [86]. This method requires two additional bulk calculations for each of the contacting insulators to locate the valence band top in each material with respect to the average potential.

The second method, which we used here, is more straightforward and is based on the analysis of the site projected partial density of states. This approach was applied for the determination of the Schottky barriers at metal-semiconductor heterojunctions [87] and it closely resembles a procedure often used in experiments. The scheme utilizes the simple fact that in the "bulk regions" of the structure, the separation of relevant "valence" energies (e.g., the Fermi energy of the metal and the valence-band maximum of the semiconductor) from core levels are the same as in the bulk. Thus, by using that energy difference along with the position of the core level in the structure $E_{c}(n)$ (where $n$ is the layer number), which essentially follows that of the average electrostatic potential in the structure, one obtains the position of the valence band energy relative to a common reference energy in the entire structure [87].

In calculations for PETN at $\mathrm{MgO}$ surface, it is natural to use the core oxygen $\mathrm{O} 2 s$ levels because oxygen atoms are present at both sides of the interface, i.e., in both crystalline PETN and $\mathrm{MgO}$. As in many oxides, the valence band top in $\mathrm{MgO}$ is derived mainly from the oxygen $p$ states [75], in PETN - mainly by hybridized oxygen and nitrogen $p$ states from the $\mathrm{NO}_{2}$ groups. The advantage of the reference potential method is in its fast convergence within a reasonably sized supercell.

The total DOS for the ideal surface together with the partial contribution from an individual PETN molecule at the $\mathrm{MgO}$ (001) surface and the DOS from a pristine $\mathrm{MgO}$ surface (without PETN molecule) are shown in Figure 4. The positions of the HOMO-LUMO of the pristine MgO surface as well as of the PETN molecule are also shown. It is clear that the energy of the PETN HOMO orbital lies significantly lower than the energy of the MgO HOMO orbital. This is consistent with the positions of the $\mathrm{O} 2$ s core levels - for $\mathrm{MgO}$ the broad core level for O 2s lies between -20 and $-18 \mathrm{eV}$ (the level is broadened because different oxide layers "feel" different average potential) while for PETN there are two apparent peaks at $-31 \mathrm{eV}$ and $-25 \mathrm{eV}$. The first 
peak corresponds to the oxygen atoms from the $\mathrm{NO}_{2}$ groups, the second peak is related to the oxygen atoms connecting $\mathrm{NO}_{2}$ groups with carbon atoms in the PETN molecule, which are positioned farther away from the interface. These numbers immediately give us an estimate for the valence band offset of about $(-12 \mathrm{eV})$. To check the accuracy of the valence band offset calculations, we added one more layer of $\mathrm{MgO}$ to the $\mathrm{MgO}$ slab and found that the offset value did not change by more than $0.3 \mathrm{eV}$, which should be considered the accuracy of these calculations.

\subsection{A PETN Molecule on an Ideal MgO (001) Surface}

The most interesting feature of the PETN - MgO interfacial electronic structure is the appearance of a new interfacial electronic transition from the $\mathrm{MgO} \mathrm{HOMO}$ orbital to the PETN LUMO level which requires only $3.42 \mathrm{eV}$ in energy (Figure 4, red curve). This transition became possible because of the band offset between the two wide band gap materials which positioned the LUMO orbital of PETN within the band gap of the MgO surface. Such a transition should transfer an electron from the substrate to the PETN molecule, which, in turn, can initiate the subsequent decomposition of the molecule. Although we did not calculate exactly the matrix element for such an interband electronic transition, an analysis of the wave functions for the $\mathrm{MgO}$ HOMO and PETN LUMO orbitals indicates that the probability of such a transition is still noticeable, i.e., a laser beam with the energy of $3.42 \mathrm{eV}$ (in the low UV frequency range) could initiate a charge transfer from the substrate to the molecules and hence significantly change the mechanism of the PETN decomposition at the surface. This transition matrix element is non-zero due to the overlap of the wave functions of an $\mathrm{O}$ atoms from $\mathrm{NO}_{2}$ groups and $\mathrm{Mg}$ atoms at the surface (Fig. 1). Although the top of the valence band in the $\mathrm{MgO}$ crystal is comprised mainly of $2 p$ oxygen states, at the surface where the $\mathrm{Mg}$ and $\mathrm{O}$ atoms are under-coordinated, some admixture of $\mathrm{Mg} s$ - and $\mathrm{Mg} p$ - states is also present, which makes it possible to contribute into the matrix element between the MgO HOMO and PETN LUMO states.

The band gap of the MgO - PETN system, $3.42 \mathrm{eV}$ (Figure 4, Table 2), is considerably lower than the values obtained for the isolated PETN molecule and the ideal $\mathrm{MgO}$ surface. For such an interface, the top of the valence band, is delocalized over the surface oxygen atoms, whereas the bottom of the conduction band is localized on the PETN nitro group, which points towards the surface. The total and projected DOS reveals that the top of the valence band is predominately 
formed from $2 p$ functions of surface oxygen atoms, whereas $2 p$ functions of the oxygen atoms of the PETN nitrogroup are mainly contributed to the bottom of the conduction band.

An analysis of Bader charges indicates a negligibly small charge transfer of $0.05 e$ from the $\mathrm{MgO}$ surface to the PETN molecule. This implies that the inter-band transition in such a system would proceed from $\mathrm{MgO}$ to PETN, and the energy of the excitation $(3.42 \mathrm{eV})$, while almost twice lower than that of each individual component ( $7.07 \mathrm{eV}$ for PETN and $5.72 \mathrm{eV}$ for the $\mathrm{MgO}$ surface) would be still too high to compare to the laser irradiation energy of $1.17 \mathrm{eV}$, observed in experiments [21-42].

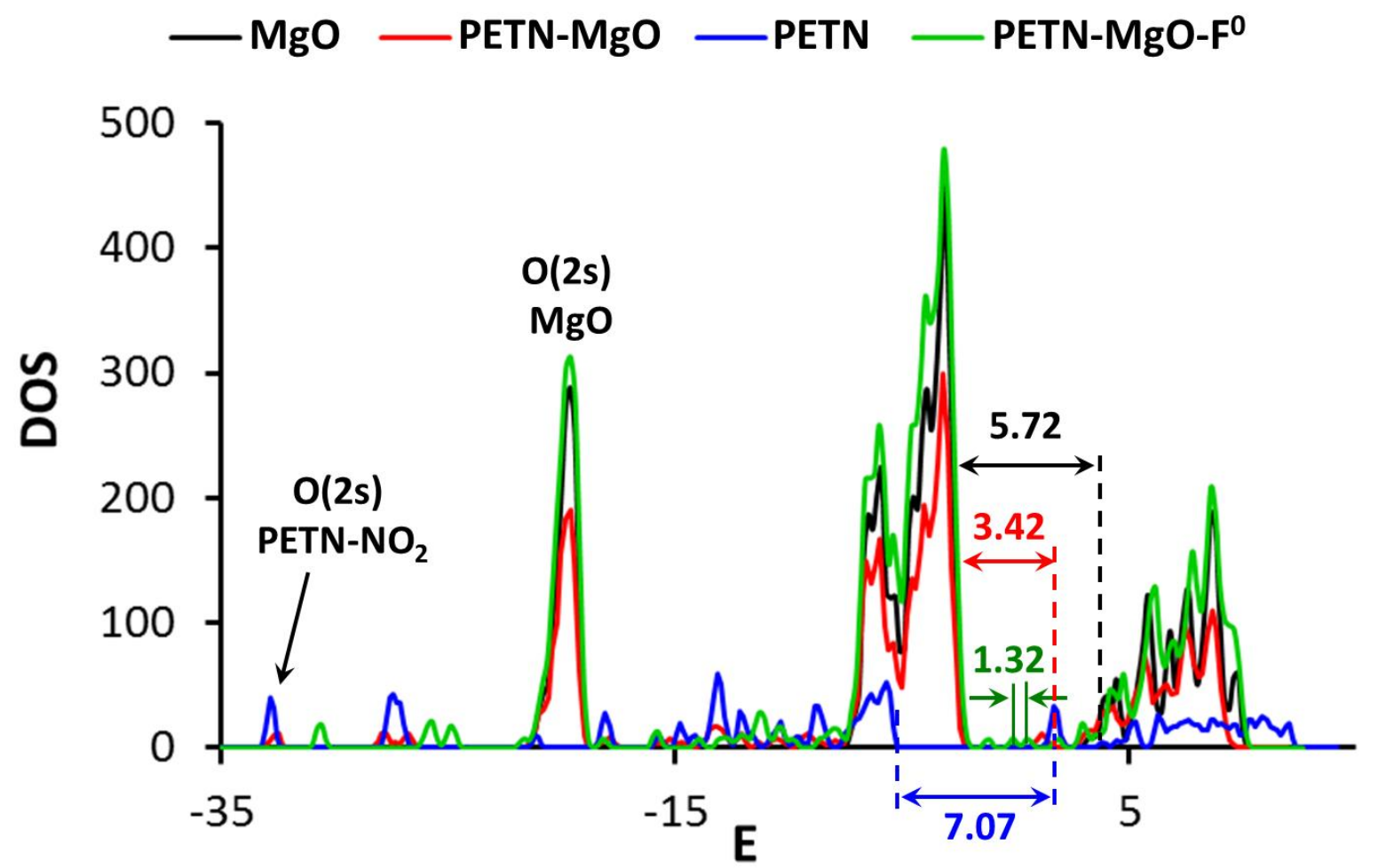

Figure 4. Total density of states of the model systems are aligned and superimposed to compare the PETN molecule adsorbed atop of the $\mathrm{F}^{0}$ center on the $\mathrm{MgO}$ surface (green line), the PETN molecule adsorbed on the ideal (defect-free) $\mathrm{MgO}$ surface (red line), the ideal $\mathrm{MgO}$ surface (black line), and the isolated PETN crystal (or molecule) (blue). The corresponding band gaps are indicated. 
We performed several additional calculations using PBE-D2 and VDW-DF functionals to reveal the effect (or the lack of thereof) of the van der Waals interactions on the electronic and geometry structures of the PETN-MgO system. The obtained results, collected in Table 3, show that the HOMO-LUMO energies calculated using PBE-D2 (1.27 eV) and VDW-DF (1.19 eV) methods are in good agreement with the HOMO-LUMO gap obtained with PBE functional (1.22 $\mathrm{eV}$ ) and that the van der Waals interactions do not significantly alter the geometry of the PETN $\mathrm{MgO}$ system as the $\mathrm{O}-\mathrm{Mg}$ distances are fairly reproduced. As expected, a noticeable difference was observed for the PETN - MgO binding energies. The binding energies calculated with the standard PBE $(0.1 \mathrm{kcal} / \mathrm{mol})$ and van-der-Waals-corrected PBE-D2 and VDW-DF functionals (8.0 and $10.0 \mathrm{kcal} / \mathrm{mol}$, respectively) differ several times. Similar trends have been recently observed for the interaction between hydroxybenzoic acids and alkali chloride surfaces [60] and for metal-organic complexes adsorbed on $\mathrm{NiO}(001)$ surface [61]. Because our paper is focused on the electronic structure rather than on binding energies, neglect of the van der Waals contribution in the Hamiltonian will not skew the accuracy of our conclusions. For our research, it is sufficient to establish the fact that the PETN molecule adsorbs on the MgO surface, as is demonstrated in our study.

Table 3. The calculated binding energies of the PETN molecule and $\mathrm{MgO}$ surface, $\mathrm{E}_{\text {bind, }}$, HOMO-LUMO energies, and distances between PETN oxygen and surface $\mathrm{Mg}$ atoms, $\mathrm{R}(\mathrm{O}-\mathrm{Mg})$.

\begin{tabular}{lccc}
\hline \hline Method & E $_{\text {bind }}, \mathrm{eV}(\mathrm{kcal} / \mathrm{mol})$ & HOMO-LUMO, eV & R(O-Mg), ^ \\
\hline \hline PBE & $0.01(0.1)$ & 1.22 & 2.278 \\
PBE-D2 & $0.35(8.0)$ & 1.27 & 2.309 \\
VDW-DF & $0.43(10.0)$ & 1.19 & 2.594 \\
\hline \hline
\end{tabular}

\subsection{A PETN Molecule on the $\mathrm{MgO}$ (001) Surface with $\mathrm{F}^{0}$-center}

The $\mathrm{F}^{0}$-center, the most common defect in many oxides, has a strong effect on both the geometric (Fig. 1d and f) and electronic structures (Fig. 4) of the PETN-MgO system. The distances between the nitrogroup's oxygen atoms (pointing towards the surface) and the $\mathrm{Mg}$ atoms become $\sim 0.2$ and $0.7 \AA$ shorter in comparison to those on the ideal MgO surface (Fig. $1 \mathrm{c}$ and d). This already demonstrates a pronounced attraction between the PETN molecule and the 
$\mathrm{F}^{0}$-center that leads to a stronger adsorption of the molecule on the surface containing vacancies than on the ideal $\mathrm{MgO}$ surface. Owing to this attractive interaction, the $\mathrm{O}-\mathrm{NO}_{2}$ bond of the PETN molecule elongates from $1.441 \AA$ on the ideal surface (which practically corresponds to the bond length of an unperturbed PETN molecule) to $2.083 \AA$ (Fig. 1c and d). Bader charges indicate that almost two electrons $(1.68 e)$ are transferred from the $\mathrm{MgO}$ surface vacancy to the PETN molecule. This additional charge is localized at the $\mathrm{O}-\mathrm{NO}_{2}$ group and explains such a large elongation of the $\mathrm{O}-\mathrm{N}$ bond. Although the obtained binding energy of the PETN molecule and the $\mathrm{F}^{0}$-center on the $\mathrm{MgO}$ surface, $1.18 \mathrm{eV}$, is underestimated as the van der Waals interactions are neglected in the total energies, it agrees with the electronic affinity of PETN molecule, 1.54 $\mathrm{eV}$ (Figure 7a). As the result, the PETN molecule transforms into a negatively charged ion, the $\mathrm{F}^{0}$-center becomes ionized and converts into an $\mathrm{F}^{2+}-$ center.

Intriguingly, the calculated band gap of the PETN-MgO system reduces to $1.32 \mathrm{eV}$ (Table 2, Fig. 4), which is notably close to $1.17 \mathrm{eV}$, the experimental energy of laser irradiation that caused the decomposition of PETN in the presence of $\mathrm{MgO}$ particles [22,41]. Unsurprisingly, perturbation of the system by the charged PETN and the $\mathrm{F}^{0}$-center is reflected in the fact that not only local defect levels (attributed to the presence of $\mathrm{F}^{0}-$ and $\mathrm{F}^{2+}$ - centers on $\mathrm{MgO}$ ) are placed in the middle of the band gap, splitting off of the valence band, but also another local level is now split off from the conduction band, as illustrated in Fig. 4. Indeed, a careful analysis of crystalline orbitals and partial DOS on atoms reveals that both the highest occupied state and the lowest unoccupied state are formed by states of the $\mathrm{F}^{0}$-center and $\mathrm{O}-\mathrm{NO}_{2}$ fragment of PETN with a strong overlap of the $2 p$ functions of the surface oxygen atoms sitting nearby the vacancy and the oxygen atoms of the $\mathrm{O}-\mathrm{NO}_{2}$ fragment of PETN (Fig. 1e-f).

The process of turning $\mathrm{F}^{0}$-center into $\mathrm{F}^{x+}$-center resembles the process of the $\mathrm{F}^{0} \rightarrow \mathrm{F}^{+}-$ photoconversion in $\mathrm{MgO}$ single crystals thermochemically reduced at elevated temperatures $[88,89,90,91]$. In crystals with an undetectable concentration of hydride ions and a moderate concentration of $\mathrm{F}^{0}$-centers, excitation with UV-light produces positively charged anion vacancies $\left(\mathrm{F}^{+}\right.$-centers $)$and electrons which are subsequently trapped at impurities. Under continuous excitation, the $\mathrm{F}^{+}$-centers release holes which are trapped at cation vacancies charge compensated by impurities. The PETN molecule adsorbed on the $\mathrm{MgO}$ surface plays a similar role as impurities on oxide crystals while at the same time enhancing the $\mathrm{F}^{0} \rightarrow \mathrm{F}^{+}$conversion process and trapping the released electrons. 


\subsection{The Charge Transfer Effect on the Stability of PETN}

It is reasonable to anticipate that dissociation mechanisms of the $[\mathrm{PETN}]^{x-}$ ion chemically bound to the $\mathrm{MgO}$ vacancy would significantly differ from the thermo-stimulated ground state chemistry, described earlier [74]. For example, a dramatic effect of charged states on decomposition chemistry, illustrated on DADNE [92] and HMX [93] molecules, was found to be manifested in reduced activation barriers, an altered dominating chemistry mechanism, and pronounced exothermicity of bond dissociation reactions.

We simulated the $\mathrm{NO}_{2}$ loss reaction, one of the most favorable thermal dissociation mechanisms, which typically occur in PETN molecules or crystals [74]. Equation (1) describes the corresponding process in which PETN molecule $\left(\mathrm{C}_{5} \mathrm{H}_{8} \mathrm{~N}_{4} \mathrm{O}_{12}\right)$, adsorbed atop of the F-center on the $\mathrm{MgO}$ surface $\left(\mathrm{MgO}\left(\mathrm{F}^{0}\right)\right)$, dissociates with $\mathrm{NO}_{2}$ radical staying on the oxide surface and the resultant residue leaving the surface. The calculated energy (which is the same here as the activation decomposition barrier) of the homolytic cleavage of the $\mathrm{O}-\mathrm{NO}_{2}$ (Fig. 1d) bond requires $10.8 \mathrm{kcal} / \mathrm{mol}$. This energy is significantly lower than $35-40 \mathrm{kcal} / \mathrm{mol}$ [74], the energy ordinarily needed for breaking such a bond in an isolated PETN molecule or PETN surface in the equilibrium ground state. Due to the extra charge gained by the PETN molecule by trapping an electron (or two) from the $\mathrm{MgO}$ vacancy, the $\mathrm{O}-\mathrm{NO}_{2}$ bond elongates to $2.083 \AA$ (Fig. 1d) to be compared to the $1.441 \AA$ in the neutral molecule (Fig. 1c) and becomes much weaker than the regular bond. An inclusion of van der Waals interactions only slightly increases the energy of O$\mathrm{NO}_{2}$ bond; the dissociation energy recalculated with PBE-D2 functional is $13.2 \mathrm{kcal} / \mathrm{mol}$.

$$
\mathrm{MgO}\left(\mathrm{F}^{0}\right)-\mathrm{C}_{5} \mathrm{H}_{8} \mathrm{~N}_{4} \mathrm{O}_{12} \rightarrow \mathrm{MgO}\left(\mathrm{F}^{0}\right)-\mathrm{NO}_{2}{ }^{\bullet}+\mathrm{C}_{5} \mathrm{H}_{8} \mathrm{~N}_{3} \mathrm{O}_{10} \bullet
$$

This modeling serves to prove that the presence of oxygen vacancies destabilizes PETN on $\mathrm{MgO}$. However, as an additional validation of whether this finding reflects the actual physical process rather than is a trivial consequence of a deficient (i.e., oversimplified) model of our system, we also simulated decomposition of the isolated charged PETN ion. Our hypothesis is that if such instability of the PETN - MgO interface is real, the parallel trends should be also valid for the isolated PETN ion in comparison to the isolated neutral molecule. In particular, the PETN molecule should be able to trap an electron and this process should reduce the decomposition barrier of the $\mathrm{NO}_{2}$ loss reaction. In the meantime, the extra charge on the PETN 
molecule does not have to be +2 , it is sufficient to show that the charged state is more energetically favorable than the equilibrium state.

These calculations were performed by GAUSSIAN code. We established that the vertical electron affinity (EA) of PETN predicted with B3LYP/6-31+G(d,p) method is 1.54 eV (Fig. 5a). The positive EA value testifies that the process of trapping an electron is energetically favorable. During the relaxation, the anion radical PETN $^{\bullet-}$ additionally gains $0.87 \mathrm{eV}$ (Fig. 5a) and finds its equilibrium configuration with one elongated $\mathrm{O}-\mathrm{NO}_{2}$ bond (Fig. 5b), which happens to be even longer than the corresponding bond in the model PETN - MgO system. The extra electron of PETN $^{\bullet-}$ (Fig. 5c) is fully localized on the distorted $\mathrm{O}-\mathrm{NO}_{2}$ fragment and predominantly formed by $2 p$ atomic functions of oxygen and nitrogen atoms. We considered two primary PETN ${ }^{\bullet-}$ decomposition mechanisms, which are most relevant to the findings of the present research (see sections 3.5-3.6) and involve the detachment of the neutral $\mathrm{NO}_{2}{ }^{\bullet}$ or anion $\mathrm{NO}_{2}{ }^{-}$species (Fig. 5a). According to our calculations, the homolytic $\mathrm{NO}_{2}{ }^{\bullet}$ loss from PETN ${ }^{\bullet-}$ requires $18.0 \mathrm{kcal} / \mathrm{mol}$ (Fig. 5a), which is in close agreement with the energy of the $\mathrm{O}-\mathrm{NO}_{2}$ bond dissociation (10.8 and $13.2 \mathrm{kcal} / \mathrm{mol}$ ) obtained for the model system PETN $-\mathrm{MgO}^{0} \mathrm{~F}^{0}$ (Eq. 1). The heterolytic detachment of the negatively charged $\mathrm{NO}_{2}{ }^{-}$from $\mathrm{PETN}^{\bullet-}$ requires $36.7 \mathrm{kcal} / \mathrm{mol}$ (Fig. 5a), which needs a higher energy than both the $\mathrm{O}-\mathrm{NO}_{2}$ bond homolysis of the negatively charged $\mathrm{PETN}^{\bullet-}$ $(18.0 \mathrm{kcal} / \mathrm{mol})$ and of the neutral PETN $(34.8 \mathrm{kcal} / \mathrm{mol})$ molecules. Hence, this mechanism is an unfavorable reaction.

Thus, those illustrative test calculations prove that the charge transfer induced by oxygen vacancies that are always present on $\mathrm{MgO}$ surfaces would destabilize the adsorbed PETN molecule. We note that the activation energy for the decomposition of PETN $-\mathrm{MgO}-\mathrm{F}^{0}$ interfaces $(10.8 \mathrm{kcal} / \mathrm{mol})$ is even somewhat lower that the laser beam energy of $1.17 \mathrm{eV}$, implying that the laser irradiation would provide an abundant perturbation of the PETN $-\mathrm{MgO}$ system to trigger its chemical decomposition. At the same time, such perturbation is not nearly sufficient to initiate regular PETN in its equilibrium state of neutral gas phase molecules, bulk crystals, or PETN surfaces, because dissociation of ground state PETN requires $35-40 \mathrm{kcal} / \mathrm{mol}$ [74].

Hence, the instability mechanism observed in laser irradiated PETN crystals with $\mathrm{MgO}$ additives is governed by charge transfer from an oxygen vacancy on the $\mathrm{MgO}$ surface to an adsorbed PETN molecule. It is clear that the efficiency of this decomposition mechanism 
depends on the concentration of vacancies (in addition to quality of crystals) and can be manipulated on demand, which opens up fascinating opportunities in the field of energetic materials and photocatalysis of organic-inorganic interfaces.
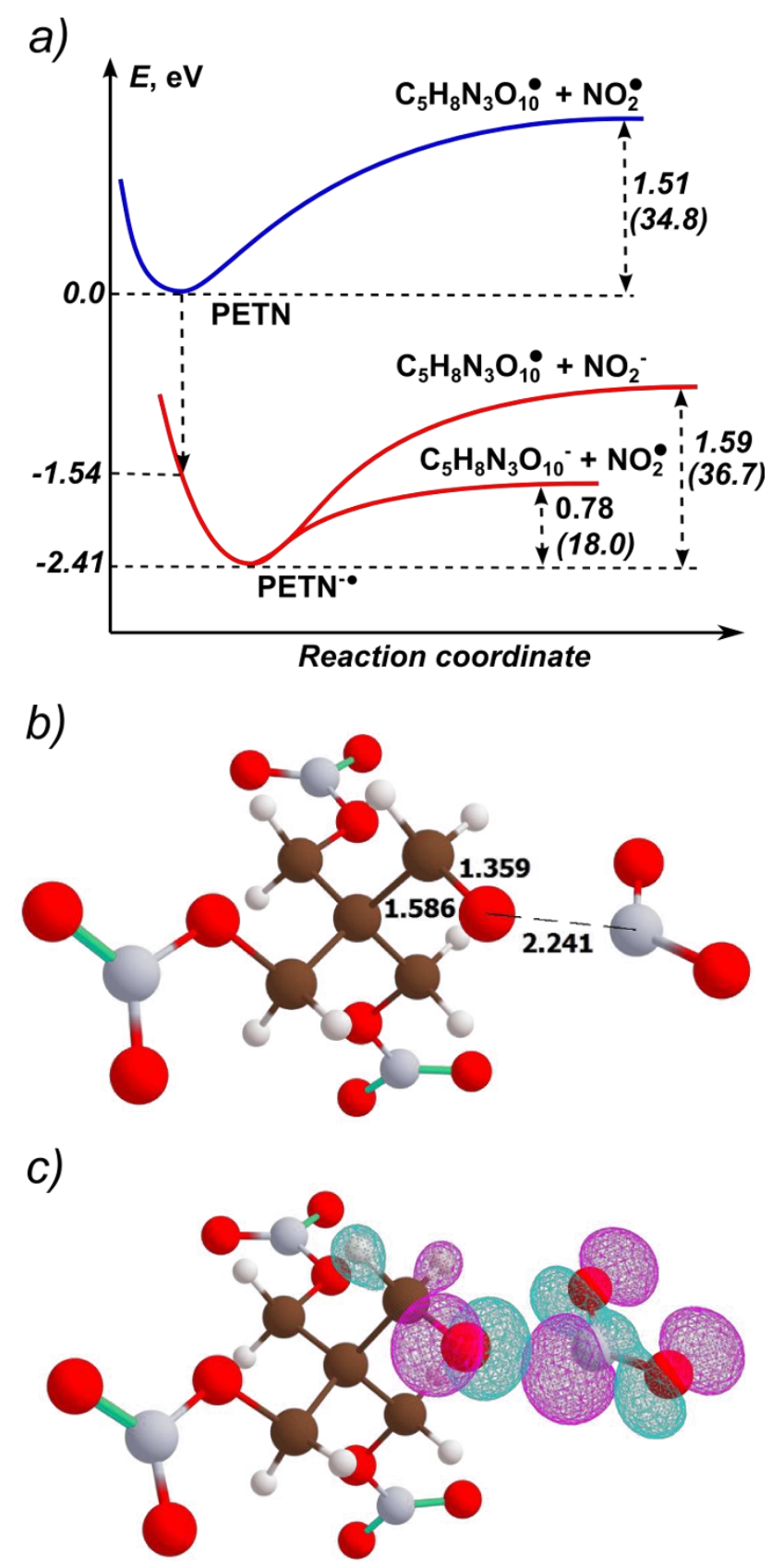

Figure 5. a) Schematic representation of fragmentation channels of neutral ground state (blue line) and negatively charged PETN molecules (red line). The numbers in parentheses are in $\mathrm{kcal} / \mathrm{mol} . \mathrm{b}$ ) The structure of PETN ${ }^{\bullet-}$ and c) The isosurface of highest occupied $\alpha$-spin molecular orbital in PETN ${ }^{\bullet}$. 


\section{SUMMARY AND CONCLUSION}

Laser initiation of energetic materials unfolds vastly new opportunities for safe handling of explosive materials and devices, the development of most effective sensors and detectors in the fight against terrorism, security screening, the removal of land-mines, finding unexploded ordnance, and for pollution monitoring. The laser initiation also provides a better control over the detonation initiation process, which enables the synthesis of new materials with superior properties, the manufacture of high precision drilling and cutting tools, and even a microsurgery in neuro-brain medicine and a stabilization of important pharmaceuticals. For the time being, a wide use of the photoinitiation of explosive decomposition reactions is at its infancy owing to the lack of general understanding of underlying fundamental mechanisms that cause laser initiation.

Recent experiments on laser initiation of PETN crystals mixed with wide band gap oxide powders demonstrated a strong dependence of the photoinitiation process upon the presence of the oxide [22]. PETN as well as most other organic nitro energetic materials are wide-band-gap dielectric crystals that are transparent to the laser light with frequencies in the visible range. Therefore, at a first glance it is difficult to grasp how a mixture of PETN with $\mathrm{MgO}$ particles may undergo decomposition under irradiation by the laser light with the energy of $1.17 \mathrm{eV}$ $[22,41]$. To unravel the effect of the $\mathrm{MgO}$ particles added to the PETN crystals on their photodecomposition and to understand interactions of the laser irradiation with the energetic material placed on the oxide substrate, we performed first principles calculations of the electronic structure of the PETN-MgO model interface and its subsequent decomposition.

We established that the electronic structure, optical properties, and chemical bond dissociation of the PETN molecule adsorbed on the (001) surface of magnesium oxide are significantly different from those of the molecular and bulk PETN. Indeed, the band gap of the PETN-MgO composite $(3.43 \mathrm{eV})$ is almost twice as low as the energy gaps of isolated PETN molecules or crystals $(\sim 6.54-7.32 \mathrm{eV})[27,43]$ and $\mathrm{MgO}$ bulk crystals or surfaces $(\sim 6.15-7.78$ eV) $[44,78]$. The top of the valence band in this system is localized on the oxygen atoms of the surface and the bottom of conduction band is formed by the oxygen atoms of the PETN nitro group.

Further, we found that the surface $\mathrm{F}^{0}$-centers, common structural defects in oxides, strongly interact with PETN and generate localized electronic levels in the band gap. As a result, the PETN molecule strongly chemically adsorbs on the oxide surface atop the vacancy. It withdraws 
almost two electrons $(1.68 e)$ from the vacancy, ionizing the vacancy and effectively generating the $\mathrm{F}^{2+}$ - center. The energy of the lowest transition reduces to $1.32 \mathrm{eV}$ and becomes remarkably close to the energy of the laser excitation of $1.17 \mathrm{eV}[22,41]$. Actually, the presence of the electronic states within the band gap contributes to the laser absorption and therefore can initiate the PETN decomposition process. The interfacial contribution to the light absorption in this system is uniquely defined by the band alignment between the two dielectrics and depends on the choice of an oxide substrate, as illustrated in Fig. 7. The charge transfer catalyzes the decomposition of PETN at the interface with the significantly reduced the activation energy barrier for primary dissociation reactions.

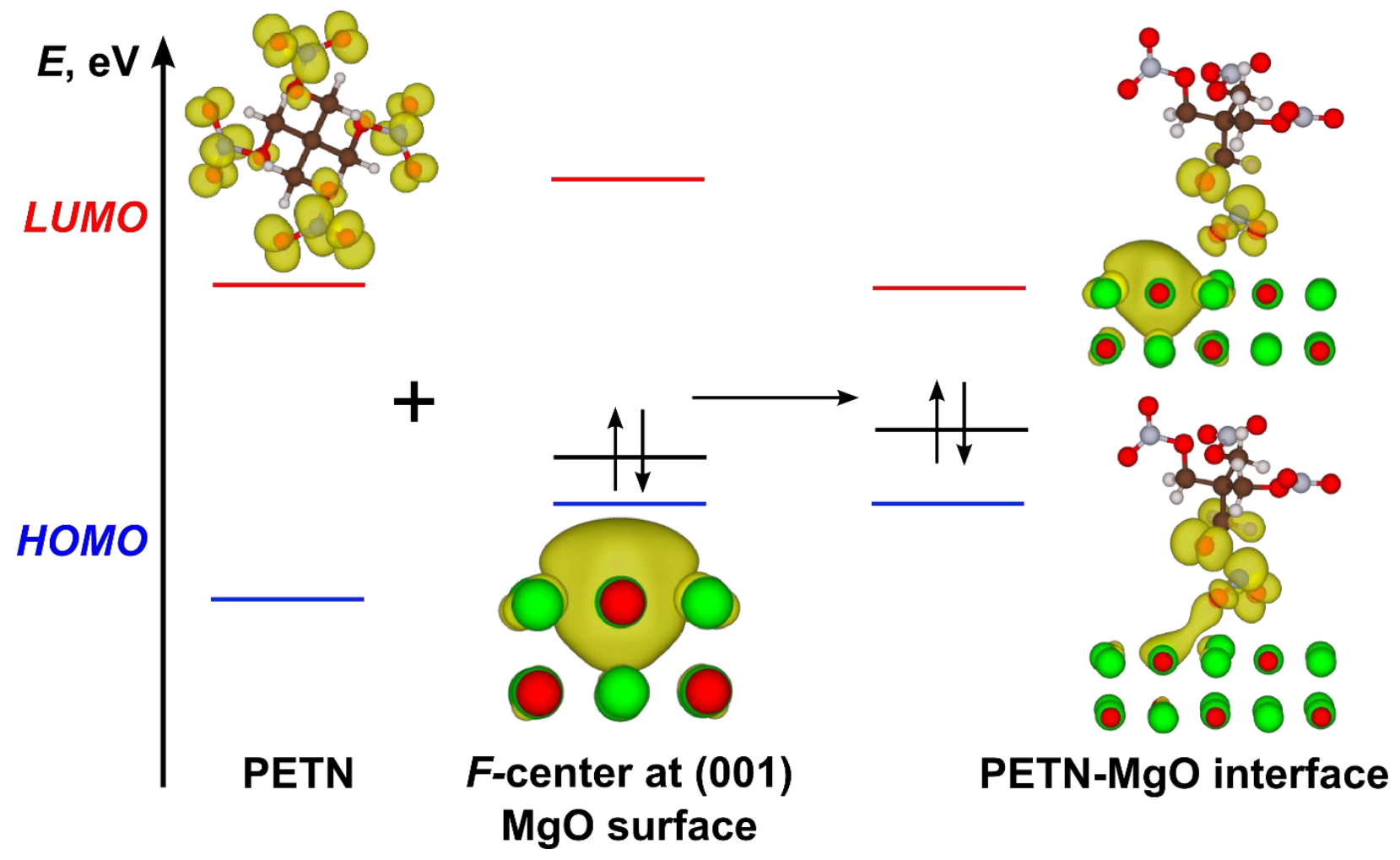

Figure 7. The band gap diagram represents how the optical absorption of individual isolated PETN and $\mathrm{MgO}$ changes at the PETN - MgO interface.

Our calculations predict that the oxygen vacancies play an imperative role in governing the laser-induced decomposition of PETN, and, most likely, of a large class of relevant nitro energetic solids. The discovered charge transfer that is facilitated at the energetic material oxide interface is instrumental in fine tuning the sensitivity of explosive material to photoinitiation. This, in turn, creates an exceptional opportunity to engineer materials interfaces that allow us to control the initiation of detonation by choosing an appropriate oxide and by 
manipulating the concentration of defects in the samples. The fine-tuning is fully attributed to an increase of the photo-sensitivity to initiation of chemistry while leaving thermo-sensitivity completely intact, which means that the thermally-stimulated reactions will not be affected. Such a high degree of explosive chemistry control was not attainable before.

The photophysics of vacancy - based defects $\left(\mathrm{F}^{0}-, \mathrm{F}^{+}-\right.$, and $\mathrm{F}^{2+}-$ centers $)$ [93-95] combined with $\mathrm{H}$ - centers $[94,95]$ or low-coordinated defect sites $[34,35,46,48]$ in oxides (steps, corners, terraces, kinks, etc) and the choice of the oxide composition [96,97] and materials' morphology (e.g., nanopowders) $[98,99,100]$ provides a playground for practically unlimited opportunities for the highly-controllable surface chemistry of molecular materials. The methodology described here could be used for any type of molecular material/wide band gap oxide dielectric interfaces. It provides a solid basis for smart design and improvement of existing molecular materials and their ingredients with targeted properties that promise to enable fundamentally new concepts of decomposition chemistry of high energy materials, energy storage and conversion, photocatalysis, and molecular electronics.

\section{AUTHOR INFORMATION}

Corresponding Author

mkukla@umd.edu or mkukla@nsf.gov

\section{Notes}

The authors declare no competing financial interest.

\section{Acknowledgements}

This research is supported in part by ONR (Grant N00014-12-1-0529) and NSF. We used NSF XSEDE resources (Grant TG-DMR-130077), the Stampede supercomputing system at TACC/UT Austin (Grant OCI-1134872), and DOE NERSC resources (Contract DE-AC0205CH11231). MMK is grateful to the Office of the Director of NSF for support under the IRD program. Any appearance of findings, conclusions, or recommendations expressed in this material are those of the author and do not necessarily reflect the views of NSF. 


\section{References}

[1] G. Seifert, Quantum molecular dynamics: The physics of explosive chemistry, Nature Physics 4 (2008) 12-13.

[2] A. Grirrane, A. Corma, H. García, Gold-Catalyzed Synthesis of Aromatic Azo Compounds from Anilines and Nitroaromatics, Science 322 (2008) 1661-1664.

[3] P. S. DeCarli, J. C. Jamieson, Formation of Diamond by Explosive Shock, Science 133 (1961) 1821-1822.

[4] N. R. Greiner, D. S. Phillips, J. D. Johnson, F. Volk, Diamonds in detonation soot, Nature 333 (1988) 440- 442.

[5] V. Koppaka, D. C. Thompson, Y. Chen, M. Ellermann, K. C. Nicolaou, R. O. Juvonen, D. Petersen, R. A. Deitrich, T. D. Hurley, V. Vasiliou, Aldehyde Dehydrogenase Inhibitors: a Comprehensive Review of the Pharmacology, Mechanism of Action, Substrate Specificity, and Clinical Application, Pharmacol. Rev. 64 (2012) 520-539.

[6] F. P. A. Fabbiani, C. R. Pulham, High-pressure studies of pharmaceutical compounds and energetic materials, Chem. Soc. Rev., 35 (2006) 932-942.

[7] Y. Bhattacharjee, New Efforts to Detect Explosives Require Advances on Many Fronts, Science 320 (2008) 1416-1415.

[8] S. S. Dasary, D. Senapati, A. K. Singh, Y. Anjaneyulu, H. Yu, P. C. Ray, Highly sensitive and selective dynamic light-scattering assay for TNT detection using p-ATP attached gold nanoparticle, ACS Appl. Mater. Interfaces, 2 (2010) 3455-3460.

[9] E. L. Rylott, R. G. Jackson, J. Edwards, G. L. Womack, H. M. B. Seth-Smith, D. A. Rathbone, S. E. Strand, N. C. Bruce, An explosive-degrading cytochrome P450 activity and its targeted application for the phytoremediation of RDX, Nature Biotechnology 24 (2006) 216 219.

[10] M. Ratner, Photochemistry: Electronic motion in DNA, Nature 397 (1999) 480-481.

[11] J. S. Levine, C. P. Rinsland, G. M. Tennille, The photochemistry of methane and carbon monoxide in the troposphere in 1950 and 1985, Nature 318 (1985) 254-257.

[12] H. B. Singh, L. J. Salas, W. Viezee, Global distribution of peroxyacetyl nitrate, Nature 321, (1986) 588-591.

[13] L. A. Pinnaduwage, A. Gehl, D. L. Hedden, G. Muralidharan, T. Thundat, R. T. Lareau, T. Sulchek, L. Manning, B. Rogers, M. Jones, J. D. Adams, Explosives: A Microsensor for Trinitrotoluene Vapour, Nature 425, (2003) 474.

[14] A. Rose, Z. Zhu, C. F. Madigan, T. M. Swager, V. Bulovic, Sensitivity Gains in Chemosensing by Lasing Action in Organic Polymers, Nature 434 (2005) 876-879

[15] T. L. Andrew, T. M. Swager, A Fluorescence Turn-On Mechanism to Detect High Explosives RDX and PETN, J. Am. Chem. Soc. 129 (2007) 7254-7255.

[16] M. N. Germain, R. L. Arechederra, S. D. Minteer, Nitroaromatic Actuation of Mitochondrial Bioelectrocatalysis for Self-Powered Explosive Sensors, J. Am. Chem. Soc. 130 (2008) 15272-15273.

[17] C. H. L. Go, W. H. Waddell, Photoinitiated autocatalytic chain decomposition of phenyl azide, J. Am. Chem. Soc. 106 (1984) 715-718.

[18] Z. Chen, S. Liu, M. Q. Yang, Y. J. Xu, Synthesis of uniform CdS nanospheres/graphene hybrid nanocomposites and their application as visible light photocatalyst for selective reduction of nitro organics in water, ACS Appl. Mater. Interfaces 5 (2013) 4309-4319. 
[19] E. Hrischlaff, R.G.W. Norrish, A Preliminary Study of the Decomposition of Nitromethane and Nitroethane, J. Chem. Soc. 1 (1936) 1580.

[20] A. N. Frolov, N. A. Kuznetsova, A. V. El'tsov, Intermolecular Photochemical Reduction of Aromatic Nitro-compounds, Russ. Chem. Rev. 45 (1976) 1024-1034.

[21] E. D. Aluker, A. G. Krechetov, A. Y. Mitrofanov, Y. P. Sakharchuk, Model of the photostimulated fragmentation of PETN molecules in selective photoinitiation, Russ. J. Phys. Chem. B 5 (2011) 821-823.

[22] E. D. Aluker, A. G. Krechetov, A. Yu Mitrofanov, D. R. Nurmukhametov, M. M. Kuklja, Laser Initiation of Energetic Materials: Selective Photoinitiation Regime in Pentaerythritol Tetranitrate, J. Phys. Chem. C 115 (2011) 6893-6901.

[23] W. Zhang, X. Ma, R. Shen, L. Wu, Y. Ye, Y. Hu, P. Zhu, Progress on Laser-Induced Decomposition of Explosives Investigated by Spectroscopic Methods. Appl. Spectrosc. Rev. 49 (2014) 550-563.

[24] E. R. Bernstein, On the Release of Stored Energy from Energetic Materials, Energetic Mater. 69 (2014) 31-52.

[25] A.V. Akimov, A. J. Neukirch, O. V. Prezhdo, Theoretical insights into photoinduced charge transfer and catalysis at metal oxide surfaces, Chem. Rev. 113 (2013) 4496-4565.

[26] A. Migani, M. J. Bearpark, M. Olivucci, M. A. Robb, Photostability versus photodegradation in the excited-state intramolecular proton transfer of nitro enamines: Competing reaction paths and conical intersections, J. Am. Chem. Soc. 129 (2007) 3703-3713.

[27] P. A. Mullen, M. K. Orloff, Ultraviolet absorption spectrum of pentaerythritol tetranitrate, J. Phys. Chem. 77 (1973) 910-911.

[28] Z. A. Dreger, Y. A. Gruzdkov, Y. M. Gupta, J. J. Dick, Shock Wave Induced Decomposition Chemistry of Pentaerythritol Tetranitrate Single Crystals: $\square$ TimeResolved Emission Spectroscopy, J. Phys. Chem. B 106 (2002) 247-256.

[29] M. W. Conroy, I. I. Oleynik, S. V. Zybin, C. T. White, First-principles investigation of anisotropic constitutive relationships in pentaerythritol tetranitrate, Phys. Rev. B 77 (2008) 094107-1-8

[30] G. Pacchioni, Quantum chemistry of oxide surfaces: From CO chemisorption to the identification of the structure and nature of point defects on MgO, Surf. Rev. Lett. 7 (2000) 277-306.

[31] G. Spoto, E. N. Gribov, G. Ricchiardi, A. Damin, D. Scarano, S. Bordiga, A. Zecchina, Carbon monoxide $\mathrm{MgO}$ from dispersed solids to single crystals: a review and new advances, Prog. Surf. Sci. 76 (2004) 71-146.

[32] P. V. Sushko, A. L. Shluger, C. Richard, A. Catlow, Relative energies of surface and defect states: ab initio calculations for the MgO (001) surface, Surf. Sci. 450 (2000) 153-170.

[33] P. V. Sushko, J. L. Gavartin, A. L. Shluger, Electronic properties of structural defects at the MgO (001) surface, J. Phys. Chem. B 106 (2002) 2269-2276.

[34] W. P. Hess, A. G. Joly, K. M. Beck, M. Henyk, Laser control of desorption through selective surface excitation, J. Phys. Chem. B 109 (2005) 19563-19578.

[35] K. P. McKenna, P. V. Sushko, A. L. Shluger, Inside powders: A theoretical model of interfaces between MgO nanocrystallites, J. Am. Chem. Soc. 129 (2007) 8600-8608.

[36] G. Pacchioni, S. Sicolo, C. Di Valentin, M. Chiesa, E. Giamello, A route toward the generation of thermally stable Au cluster anions supported on the $\mathrm{MgO}$ surface, J. Am. Chem. Soc. 130 (2008) 8690-8695. 
[37] M. Sterrer, E. Fischbach, T. Risse, H. J. Freund, Geometric characterization of a singly charged oxygen vacancy on a single-crystalline $\mathrm{MgO}(001)$ film by electron paramagnetic resonance spectroscopy, Phys. Rev. Lett. 94 (2005) 186101.

[38] K. M. Neyman, C. Inntam, A. V. Matveev, V. A. Nasluzov, N. Rösch, Single d-metal atoms on Fs and Fs+ defects of $\mathrm{MgO}$ (001): A theoretical study across the periodic table, J. Am. Chem. Soc. 127 (2005) 11652-11660.

[39] Z. Yan, S. Chinta, A. A. Mohamed, J. P. Fackler, D. W. Goodman, The role of F-centers in catalysis by Au supported on MgO. J. Am. Chem. Soc. 127 (2005) 1604-1605.

[40] P. Tian, X. Y. Han, G. L. Ning, H. X. Fang, J. W. Ye, W. T. Gong, Y. Lin, Synthesis of Porous Hierarchical $\mathrm{MgO}$ and Its Superb Adsorption Properties, ACS Appl. Mater. Interfaces, 5 (2013) 12411-12418.

[41] E. D. Aluker, A. G. Krechetov, A. Yu. Mitrofanov, A. S. Zverev, M. M. Kuklja, Understanding Limits of the Thermal Mechanism of Laser Initiation of Energetic Materials, J. Phys. Chem. C 116 (2012) 24482-24486.

[42] E. D. Aluker, A. G. Krechetov, A. Y. Mitrofanov, A. S. Zverev, M. M. Kuklja, Topography of Photochemical Initiation in Molecular Materials, Molecules 18 (2013) 14148-14160.

[43] R. V. Tsyshevsky, O. Sharia, M. M. Kuklja, Energies of Electronic Transitions of PETN Molecules and Crystals, J. Phys. Chem. C 118 (2014) 9324-9335.

[44] R. C. Whited, W. C. Walker, Exciton Spectra of $\mathrm{CaO}$ and MgO, Phys. Rev. Lett. 22 (1969) $1428-1430$.

[45] M. Roessler, W. C. Walker, Electronic Spectrum and Ultraviolet Optical Properties of Crystalline MgO, Phys. Rev. 159 (1967) 733-738.

[46] W. P. Hess, A. G. Joly, K. M. Beck, M. Henyk, P. V. Sushko, P. E. Trevisanutto, A. L. Shluger, Laser Control of Desorption through Selective Surface Excitation, J. Phys. Chem. B 109 (2005) 19563-19578.

[47] M. Sterrer, O. Diwald, E. Knözinger, P. V. Sushko, A. L. Shluger, Energies and Dynamics of Photoinduced Electron and Hole Processes on MgO Powders, J. Phys. Chem. B 106 (2002) 12478-12482.

[48] A. Zecchina, M. G. Lofthouse, F. S. Stone, Reflectance Spectra of Surface States in Magnesium Oxide and Calcium Oxide, J. Chem. Soc. Faraday Trans. I 71 (1975) 1476-1490.

[49] S. Coluccia, A. M. Deane, A. J. Tench, Photoluminescent Spectra of Surface States in Alkaline Earth Oxides, J. Chem. Soc. Faraday Trans. I 74 (1978) 2913-2922.

[50] O. Diwald, M. Sterrer, E. Knözinger, P. V. Sushko, A. L. Shluger, Wavelength selective excitation of surface oxygen anions on highly dispersed $\mathrm{MgO}$, J. Chem. Phys. 116 (2002) 1707-1712.

[51] P. Hohenberg, W. Kohn, Inhomogeneous electron gas, Phys. Rev. 136 (1964) B864-B71.

[52] W Kohn, L. J. Sham, Self-consistent equations including exchange and correlation effects, Phys. Rev. A 140 (1965) A1133-A38.

[53] J. P. Perdew, K. Burke, M. Ernzerhof, Generalized gradient approximation made simple, Phys. Rev. Lett. 77 (1996) 3865-3868.

[54] C. Adamo, V. Barone, Toward reliable density functional methods without adjustable parameters: The PBE0 model, J. Chem. Phys. 110 (1999) 6158-6169.

[55] G. Kresse, J. Futhmuller, Efficiency of ab-initio total energy calculations for metals and semiconductors using a plane-wave basis set, Comput. Mater. Sci. 6 (1996) 15-50. 
[56] G. Kresse, F. Furthmuller, Efficient iterative schemes for ab initio total-energy calculations using a plane-wave basis set, Phys. Rev. B 54 (1996) 11169-11186.

[57] G. Kresse, J. Hafner, Ab initio molecular dynamics for liquid metals, Phys. Rev. B 47 (1993) 558-561.

[58] P. E. Blöchl, Projector augmented-wave method, Phys. Rev. B 50 (1994) 17953-17979.

[59] H. H Cady, A. C. Larson, Pentaerythritol tetranitrate II: its crystal structure and transformation to PETN I; an algorithm for refinement of crystal structures with poor data. Acta Cryst. B 31 (1975) 1864-1869.

[60 ] Wei Chen, Christoph Tegenkamp, Herbert Pfnur, and Thomas Bredow. Insight from FirstPrinciples Calculations into the Interactions between Hydroxybenzoic Acids and Alkali Chloride Surfaces, J. Phys. Chem. C 114 (2010) 460-467.

[61] Alexander Schwarz, David Z. Gao, Knud Lammle, Josef Grenz, Matthew B. Watkins, Alexander L. Shluger, and Roland Wiesendanger. Determining Adsorption Geometry, Bonding, and Translational Pathways of a Metal-Organic Complex on an Oxide Surface: Co-Salen on $\mathrm{NiO}(001)$, J. Phys. Chem. C 117 (2013) 1105-1112.

[62] S. Grimme, Semiempirical GGA-type density functional constructed with a long-range dispersion correction, J. Comp. Chem. 27 (2006) 1787-1799.

[63] M. Dion, H. Rydberg, E. Schroder, D. C. Langreth, B. I. Lundqvist, Van der Waals Density Functional for General Geometries, Phys. Rev. Lett. 92 (2004) 246401-1-4.

[64] T. Thonhauser, V. R. Cooper, S. Li, A. Puzder, P. Hyldgaard, and D. C. Langreth, Van der Waals density functional: Self-consistent potential and the nature of the van der Waals bond, Phys. Rev. B 76, (2007) 125112.

[65] G. Roman-Perez and J. M. Soler, Efficient Implementation of a van der Waals Density Functional: Application to Double-Wall Carbon Nanotubes, Phys. Rev. Lett., 103, (2009) 096102.

[66] P. Giannozzi, S. Baroni, N. Bonini, M. Calandra, R. Car, C. Cavazzoni, D. Ceresoli, G. L. Chiarotti, M. Cococcioni, I. Dabo, A. D. Corso, S. de Gironcoli, S. Fabris, G. Fratesi, R. Gebauer, U. Gerstmann, C. Gougoussis, A. Kokalj, M. Lazzeri, L. Martin-Samos, N. Marzari, F. Mauri, R. Mazzarello, S. Paolini, A. Pasquarello, L. Paulatto, C. Sbraccia, S. Scandolo, G. Sclauzero, A. P. Seitsonen, A. Smogunov, P. Umari, R. M. Wentzcovitch, QUANTUM ESPRESSO: a modular and open-source software project for quantum simulations of materials, J. Phys.:Condens. Matter. 21 (2009) 395502.

[67] A. D. Becke, Density-functional thermochemistry. III. The role of exact exchange, J. Chem. Phys., 98 (1993) 5648-5652.

[68] Gaussian 09, Revision B.01, M. J. Frisch, G. W. Trucks, H. B. Schlegel, G. E. Scuseria, M. A. Robb, J. R. Cheeseman, G. Scalmani, V. Barone, B. Mennucci, G. A. Petersson, H. Nakatsuji, M. Caricato, X. Li, H. P. Hratchian, A. F. Izmaylov, J. Bloino, G. Zheng, J. L. Sonnenberg, M. Hada, M. Ehara, K. Toyota, R. Fukuda, J. Hasegawa, M. Ishida, T. Nakajima, Y. Honda, O. Kitao, H. Nakai, T. Vreven, J. A. Montgomery, Jr., J. E. Peralta, F. Ogliaro, M. Bearpark, J. J. Heyd, E. Brothers, K. N. Kudin, V. N. Staroverov, R. Kobayashi, J. Normand, K. Raghavachari, A. Rendell, J. C. Burant, S. S. Iyengar, J. Tomasi, M. Cossi, N. Rega, J. M. Millam, M. Klene, J. E. Knox, J. B. Cross, V. Bakken, C. Adamo, J. Jaramillo, R. Gomperts, R. E. Stratmann, O. Yazyev, A. J. Austin, R. Cammi, C. Pomelli, J. W. Ochterski, R. L. Martin, K. Morokuma, V. G. Zakrzewski, G. A. Voth, P. Salvador, J. J. Dannenberg, S. Dapprich, A. D. Daniels, Ö. Farkas, J. B. Foresman, J. V. Ortiz, J. Cioslowski, and D. J. Fox, Gaussian, Inc., Wallingford CT, 2009. 
[69] J. A. McLeod, E. Z. Kurmaev, P. V. Sushko, T. D. Boyko, I. A. Levitsky, A. Moewes, Selective Response of Mesoporous Silicon to Adsorbants with Nitro Groups, Chem. Eur. J. 18 (2012) $2912-2922$.

[70] D. C. Sorescu, J. A. Boatz, D. L. Thompson, First-Principles Calculations of the Adsorption of Nitromethane and 1,1-Diamino-2,2-dinitroethylene (FOX-7) Molecules on the $\mathrm{Al}(111)$ Surface, J. Phys. Chem. B 107 (2003) 8953-8964.

[71] D. C. Sorescu, J. A. Boatz, D. L. Thompson, First-Principles Calculations of the Adsorption of Nitromethane and 1,1-Diamino-2,2-dinitroethylene (FOX-7) Molecules on the r$\mathrm{Al}_{2} \mathrm{O}_{3}(0001)$ Surface, J. Phys. Chem. B 109 (2005) 1451-1463

[72] Zepeda-Ruiz, L. A.; Maiti, A.; Gee, R.; Gilmer, G. H.; Weeks, B. L. Size and Habit

Evolution of PETN Crystals： A Lattice Monte Carlo Study. J. Cryst. Growth 291 (2006) 461-

467.

[73] M. M. Kuklja, A. B. Kunz, Electronic structure of molecular crystals containing edge dislocations, J. Appl. Phys. 89 (2001) 4962-4970.

[74] R. Tsyshevsky, O. Sharia, M. M. Kuklja, Thermal Decomposition Mechanisms of Nitroesters: Ab Initio Modeling of Pentaerythritol Tetranitrate, J. Phys. Chem. C 117 (2013) 18144-18153.

[75] P. D. C. King, T. D. Veal, A. J. Schleife, Zúñiga-Pérez, B. Martel, P. H. Jefferson, F. Fuchs, V. Muñoz-Sanjosé, F. Bechstedt, C. F. McConville, Valence-band electronic structure of CdO, $\mathrm{ZnO}$, and $\mathrm{MgO}$ from X-ray photoemission spectroscopy and quasi-particle-corrected densityfunctional theory calculations, Phys. Rev. B 79 (2009) 205205-1-6.

[76] E. S. Gaffney, T. J. Ahrens, Heat of Formation of $\mathrm{O}^{2-}$, J. Chem. Phys. 51 (1969) 1088-1091.

[77] M. Causa, R. Dovesi, C. Pisani, C. Roetti, Electronic structure and stability of different crystal phases of magnesium oxide, Phys. Rev B 33 (1986) 1308-1316.

[78] P. A. Cox, A. A. Williams, Surface excitons on ionic crystals. Surf. Sci. Lett. 175 (1986) L782-L786.

[79] A. L Shluger, L. N. Kantorovich, A. I. Livshits, M. J. Gillan, Ionic and electronic processes at ionic surfaces induced by atomic-force-microscope tips, Phys. Rev. B. 56 (1997) 1533215344.

[80] I. Tanaka, F. Oba, K. Tatsumi, M. Kunisu, M. Nakano, H. Adachi, Theoretical formation energy of oxygen-vacancies in oxides, Mater. Trans. 43 (2002) 1426-1429.

[81] A. M. Ferrari, G. Pacchioni, Electronic Structure of F and V Centers on the MgO Surface, J. Phys. Chem. 99 (1995) 17010-17018.

[82] W. Schottky, Vereinfachte und erweiterte Theorie der Randschicht-gleichrichter, Z. Physik 118 (1942) 539-592.

[83] M. Peressi, N. Binggeli, A. Baldereschi, Band engineering at interfaces: theory and numerical experiments, J. Phys. D 31 (1998) 1273.

[84] J. Tersoff, Band Lineups at II-VI Heterojunctions: Failure of the Common-Anion Rule, Phys. Rev. Lett. 56 (1986) 2755.

[85] D. M. Bylander, L. Kleinman, Ab initio (GaAs) $)_{3}(\mathrm{AlAs})_{3}(001)$ superlattice calculations: Band offsets and formation enthalpy, Phys. Rev. B 36 (1987) 3229-3236.

[86] C. G. Van de Walle, R. M. Martin, "Absolute', deformation potentials: Formulation and ab initio calculations for semiconductors, Phys. Rev. Lett. 62 (1989) 2028-2031.

[87] S. N. Rashkeev, W. R. L. Lambrecht, B. Segall, Electronic structure, Schottky barrier, and optical spectra of the SiC/TiC $\{111\}$ interface, Phys. Rev. B 55 (1997) 16472-16468. 
[88] R. González, M. A. Monge, J. M. Santiuste, R. Pareja, Y. Chen, E. Kotomin, M. M. Kukla, A. I. Popov, Photoconversion of F-type centers in thermochemically reduced $\mathrm{MgO}$ single crystals, Phys. Rev. B 59 (1999) 4786-4790.

[89] E. A. Kotomin, M. M. Kuklja, R. I. Eglitis, A. I. Popov, Quantum chemical simulations of the optical properties and diffusion of electron centres in MgO crystals, Mater. Sci. Eng. B 37 (1996) 212-214.

[90] R. I. Eglitis, M. M. Kuklja, E. A. Kotomin, A. Stashans, A. I. Popov, Semi-empirical simulations of the electron centers in MgO crystal, Comput. Mater. Sci. 5 (1996) 298-306.

[91] A. I. Popov, E. A. Kotomin, M. M. Kuklja, Quantum chemical calculations of the electron center diffusion in MgO crystals, Phys. Stat. Sol. (B) 195 (1996) 61-66.

[92] A. V. Kimmel, P. V. Sushko, A. L. Shluger, M. M. Kuklja, Effect of charged and excited states on the decomposition of 1,1-diamino-2,2-dinitroethylene molecules, J. Chem. Phys. 126 (2007) 234711-234711-10.

[93] M. M. Kuklja, Quantum-Chemical Modeling of Energetic Materials: Chemical Reactions Triggered by Defects, Deformations, and Electronic Excitations, Adv. Quant. Chem. 68 (2014) 71-146.

[94] M. M. Kuklja, E. V. Stefanovich, E. A. Kotomin, A. I. Popov, R. González, Y. Chen, Ab initio and semiempirical calculations of H-centers in MgO crystals, Phys. Rev. B, 59 (1999) 1885-1890.

[95] M. A. Monge, R. González, A. I. Popov, R. Pareja, Y. Chen, E. A. Kotomin, M. M. Kuklja, The dynamics of the hydride ion in MgO single crystals, Defect Diffus. Forum 169 (1999) 112.

[96] M. Pesci, F. Gallino, C. Di Valentin, G. Pacchioni, Nature of Defect States in NitrogenDoped MgO, J. Phys. Chem. C 114 (2009) 1350-1356.

[97] C. Di Valentin, G. Pacchioni, Spectroscopic Properties of Doped and Defective Semiconducting Oxides from Hybrid Density Functional Calculations, Acc. Chem. Res. 39 (2014) 861-867.

[98] R. W. Conner, D. D. Dlott, Ultrafast Condensed-Phase Emission From Energetic Composites of Teflon and Nanoaluminum. J. Phys. Chem. A 114 (2010) 6731-6741.

[99] K. Kappagantula, M. L. Pantoya, E. M. Hunt, Impact ignition of aluminum-teflon based energetic materials impregnated with nano-structured carbon additives, J. Appl. Phys. 112 (2012) 024902-1-7.

[100] X. Zheng, A. D. Curtis, W. L. Shaw, D. D. Dlott, Shock initiation of nano-Al+ Teflon: time-resolved emission studies, J. Phys. Chem. C 117 (2013) 4866-4875. 
Table of Content image

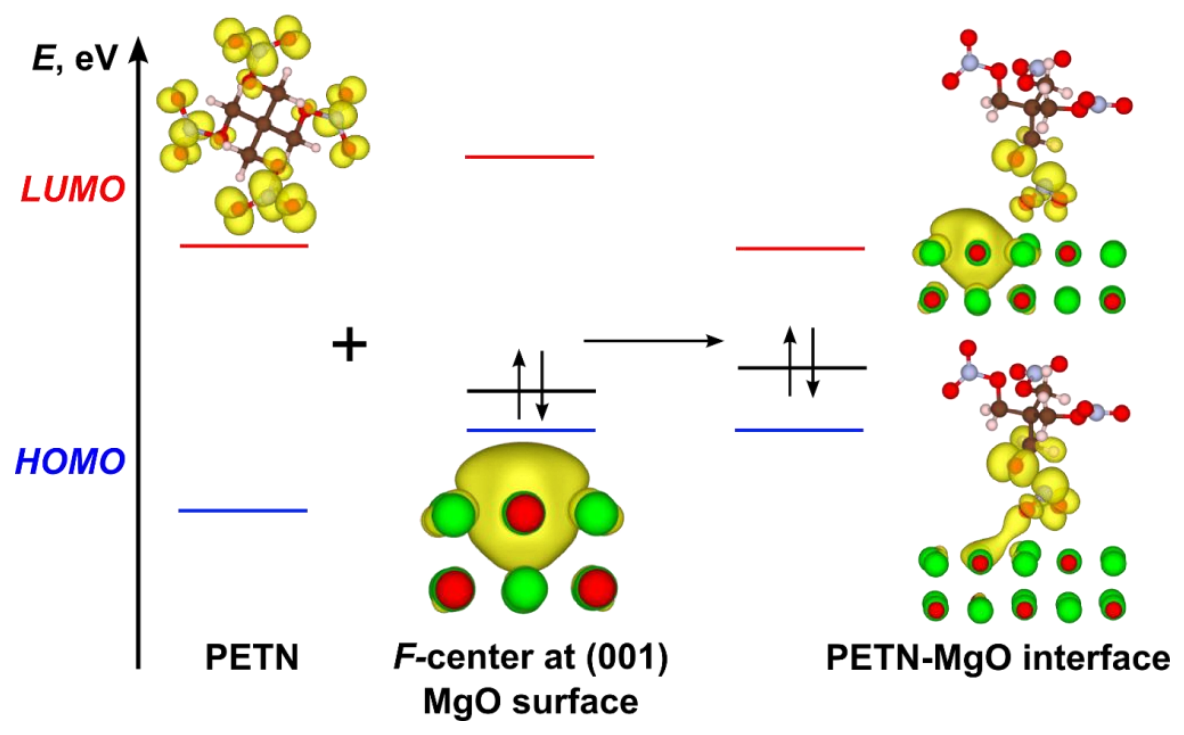

